\title{
Photonuclear Effect in Heavy Deformed Nuclei*
}

\author{
HARTMUTh ARENHÖVEL \\ Institut für theoretische Physik, Universität Frankfurt/Main, Frankfurt/Main, Germany \\ AND \\ Michael Danos \\ National Bureau of Standards, Washington, D. C. \\ AND \\ WALTER Greiner \\ Institut für theoretische Physik, Universität Frankfurt/Main, Frankfurt/Main, Germany \\ and \\ National Bureau of Standards, Washington, D. C.
}

(Received 12 October 1966)

\begin{abstract}
The theory of Raman scattering is extended to include electric-quadrupole radiation. The results obtained are used to compute the elastic and Raman scattering cross sections of heavy deformed nuclei. The dipole and quadrupole resonances are described by a previously developed theory which includes surface vibrations and rotations. The computed cross sections are compared with experimental data for all those nuclei where both absorption and scattering cross sections are available. Some discrepances still exist in certain details; however, the over-all agreement between theory and experiment is very good.
\end{abstract}

\section{INTRODUCTION}

$T$ HE present paper has two parts. In the first part, the theory of elastic and Raman scattering is extended to quadrupole radiation including dipolequadrupole interference. Together with the well-known results for dipole radiation, ${ }^{1,2}$ the formulas obtained are used to compute the photon-scattering cross sections on the basis of the dynamic collective theory ${ }^{3-6}$ of the giant resonance in heavy deformed nuclei. In the second part, we attempt to obtain as complete as possible theoretical fits to the presently available high-resolution experiments, taking together both absorption and scattering data. In other words, our aim is to determine how consistent is the totality of the information concerning the nuclear giant resonance.

We believe that this is the correct time for such an attempt. The development of both theory and experiment of the photonuclear effect over the last 15 years has led from a qualitative picture to a quantitative description. ${ }^{7}$ In other words, the qualitative features are quite well understood. The open questions are of a quantitative nature; e.g., what fraction of the oscillator

* Work supported in part by the Deutsche Forschungsgemeinschaft with a contract on nuclear-structure studies, and by the Deutsches Forschungsministerium.

${ }_{1}$ G. Placzek, in Marx Handbuch der Radiologie 6, 2 (1934); 6, 205 (1934).

2 U. Fano, National Bureau of Standards (U. S.) Technical Note 83, 1960 (unpublished).

${ }^{3}$ M. Danos and W. Greiner, Phys. Rev. 134, B284 (1964).

${ }^{4}$ M. Danos, W. Greiner, and C. B. Kohr, Phys. Rev. 138, B1055 (1965).

${ }^{5}$ M. Danos, W. Greiner, and C. B. Kohr, Phys. Rev. 151, 761 (1966).

${ }^{6}$ R. Ligensa, W. Greiner, and M. Danos, Phys. Rev. Letters 16, 363 (1966).

${ }^{7}$ M. Danos and E. G. Fuller, Ann. Rev. Nucl. Sci. 15, 29 (1965). strength is associated with the giant resonance, or, to what accuracy can the shape of the cross section be quantitatively described, etc. It will be seen that very few nuclei have been investigated with a sufficient accuracy for an analysis of the kind attempted here. It would also be exceedingly desirable to have available high-quality data concerning nuclei from the transition region, i.e., from the region where the deformations become small. Examples of such nuclei are neodymium, samarium, and osmium. The data should include the region above $20 \mathrm{MeV}$, i.e., the location of the giant quadrupole resonance..$^{5,6}$

The theoretical part of the paper is contained in Secs. II through IV. The formulas for the electric quadrupole elastic and Raman scattering including interference with dipole radiation are developed in Sec. II. The scattering amplitudes are given in terms of reduced matrix elements of the multipole operators. The final formulas for the diverse scattering cross sections are worked out for nonaligned targets. In a description of experiments performed with aligned targets, ${ }^{8}$ one would have to use directly the expressions for the scattering amplitudes. A résumé of the nuclear theory is given in Sec. III, and the reduced matrix elements needed in the formulas for the different cross sections are evaluated in Sec. IV. The second part of the paper, i.e., the detailed comparison between the experimental data and the theoretical predictions, is contained in Sec. V. We have analyzed the data of all cases where both absorption and scattering cross sections, i.e., a complete set of data, are available. Section VI contains a summary and a discussion of the results obtained.

${ }^{8}$ E. Ambler, E. G. Fuller, and H. Marshak, Phys. Rev. 138, B117 (1965).

1109 


\section{SCATTERING AND ABSORPTION CROSS-SECTION FORMULAS}

The transition probability for the scattering of incoming photons with wave vector $k$ into outgoing photons $k^{\prime}$ is given by ${ }^{9}$

the nuclear state changing from $i$ to $f$.

$$
d \sigma / d \Omega=\left(k^{\prime} / k\right)\left|K_{i f}\right|^{2},
$$

We devote this section to the description of the matrix elements $K_{i f}$. We begin by developing the general formula for arbitrary photon multipolarity. Later we specialize to the case of dipole and quadrupole scattering. In general we have

$$
\begin{aligned}
K_{i f}=-\frac{Z^{2} e^{2}}{A M c^{2}} \mathbf{e}_{\mathbf{k}^{\prime} \lambda^{\prime}}{ }^{*} \cdot \mathbf{e}_{\mathbf{k} \lambda} \delta_{i f}+\frac{1}{c^{2}} \sum_{n}\left[\left\langle f\left|\int \mathbf{j} \cdot \mathbf{A}^{*}\left(\mathbf{k}^{\prime}\right) \frac{1}{H-E-i \eta} \int \mathbf{j} \cdot A(\mathbf{k})\right| i\right\rangle\right. & \\
& \left.+\left\langle f\left|\int \mathbf{j} \cdot \mathbf{A}(\mathbf{k}) \frac{1}{H+E^{\prime}+i \eta} \int \mathbf{j} \cdot \mathbf{A}^{*}\left(\mathbf{k}^{\prime}\right)\right| i\right\rangle\right] .
\end{aligned}
$$

In this equation $\mathbf{e}_{\mathrm{k}^{\prime} \lambda^{\prime}}$ and $\mathbf{e}_{\mathrm{k} \lambda}$ are the polarization unit vectors of the photons for circular polarization, $E$ is the incoming photon energy, $H$ is the nuclear Hamiltonian normalized so that the nuclear ground-state energy equals zero, and

$$
\mathbf{A}(\mathbf{k})=\mathbf{e}_{\mathbf{k} \lambda} e^{i \mathbf{k} \cdot \mathbf{r}}
$$

is the vector potential. The imaginary part $\eta$ of the energy denominators is to be taken in the limit $\eta-0$.

In Appendix B of Ref. 10 it was shown that under certain circumstances the absorption cross section of a damped giant resonance state $|\mathrm{GR}\rangle$ can be approximated by a Lorentz line, i.e., that it is described by the forward scattering amplitude $f(E)$ which has an imaginary part given by

$$
\begin{aligned}
\operatorname{Im} f(E) & =\frac{1}{c^{2}}\left[\left\langle 0\left|\int \mathbf{j} \cdot \mathbf{A}^{*}(\mathbf{k})\right| \mathrm{GR}\right\rangle \frac{\Gamma / 2}{\left(E-\epsilon_{\mathrm{GR}}\right)^{2}+\Gamma^{2} / 4}\left\langle\mathrm{GR}\left|\int \mathbf{j} \cdot \mathbf{A}(\mathbf{k})\right| 0\right\rangle\right. \\
& \left.-\left\langle 0\left|\int \mathbf{j} \cdot \mathbf{A}(\mathbf{k})\right| \mathrm{GR}\right\rangle \frac{\Gamma / 2}{\left(E+\epsilon_{\mathrm{GR}}\right)^{2}+\Gamma^{2} / 4}\left\langle\mathrm{GR}\left|\int \mathbf{j} \cdot \mathbf{A}(\mathbf{k})\right| 0\right\rangle\right] \\
& =\frac{1}{2 c^{2}}\left|\left\langle 0\left|\int \mathbf{j} \cdot \mathbf{A}(\mathbf{k})\right| \mathrm{GR}\right\rangle\right|^{2} \frac{\Gamma \epsilon_{\mathrm{GR}} E}{\left(E^{2}-E_{\mathrm{GR}}\right)^{2}+E^{2} \Gamma^{2}}, \\
E_{\mathrm{GR}^{2}} & =\epsilon_{\mathrm{GR}^{2}}{ }^{2}+\Gamma^{2} / 4 .
\end{aligned}
$$

One sees that the same imaginary part is obtained if in (2) one replaces formally the eigenstates $|n\rangle$ of the nuclear Hamiltonian by the giant resonance state $|G R\rangle$ and introduces a finite width $\Gamma$ which formally replaces $\eta$ in the energy denominators.

With these substitutions, we thus have

$$
\operatorname{Im} f(E)=\operatorname{Im} K(E) .
$$

Then from the dispersion relations it follows that also the real parts of $K(E)$ and $f(E)$ are equal if $\operatorname{Re} K(0)=\operatorname{Re} f(0)$. This is so because the real part of the scattering amplitude at zero energy is given by the Thomson amplitude in both cases. We emphasize that the above replacements are only approximately valid and refer to Ref. 10 for a discussion of the limits of validity. If several giant resonances are present, which is the case in heavy nuclei, then their contributions to the scattering amplitude add. Thus we finally have for the complete scattering amplitude

$$
\begin{aligned}
K_{i f}=-\frac{Z^{2} e^{2}}{A M c^{2}} \mathbf{e}_{\mathbf{k}^{\prime} \lambda^{\prime}} * \mathbf{e}_{\mathbf{k} \lambda} \delta_{i f}+\sum_{n}\left[\left\langle f\left|\int \mathbf{j} \cdot \mathbf{A}^{*}\left(\mathbf{k}^{\prime}\right)\right| \mathrm{GR}_{n}\right\rangle \frac{1}{E_{\mathrm{GR}_{n}}-E-\frac{1}{2} i \Gamma_{n}}\left\langle\mathrm{GR}_{n}\left|\int \mathbf{j} \cdot \mathbf{A}(\mathbf{k})\right| i\right\rangle\right. & \\
& \left.+\left\langle f\left|\int \mathbf{j} \cdot \mathbf{A}(\mathbf{k})\right| \mathrm{GR}_{n}\right\rangle \frac{1}{E_{\mathrm{GR}_{n}}+E^{\prime}+\frac{1}{2} i \Gamma_{n}}\left\langle\mathrm{GR}_{n}\left|\int \mathbf{j} \cdot \mathbf{A}^{*}\left(\mathbf{k}^{\prime}\right)\right| i\right\rangle\right] .
\end{aligned}
$$

\footnotetext{
${ }^{9}$ W. Heitler, The Quantum Theory of Radiation (Clarendon Press, Oxford, England, 1954).
}

${ }^{10}$ M. Danos and W. Greiner, Phys. Rev. 138, B87.6 (1965). 
In the following we shall drop the notation $\left|\mathrm{GR}_{n}\right\rangle$ and replace it by $|n\rangle$, where $n$ now stands for the state of the $n$th giant resonance.

We now perform a multipole expansion of the vector potential, ${ }^{11}$ i.e.,

$$
\begin{aligned}
& \mathbf{A}^{*}\left(\mathbf{k}^{\prime}\right)=\mathbf{e}_{p}{ }^{*} e^{-i \mathbf{k}^{\prime} \cdot \mathbf{r}}=(2 \pi)^{1 / 2} \sum_{L, M}(-)^{L-p L}(2 L+1)^{1 / 2} \mathbf{B}_{L M^{k^{\prime} p} D_{M,-p} L} \\
& \mathbf{B}_{L M^{k^{\prime} p}}=p \mathbf{A}_{L M^{k^{\prime}}}(m)+i \mathbf{A}_{L M^{k^{\prime}}}(e)
\end{aligned}
$$

which is an expansion in multipole fields $\mathbf{A}_{L M}$ defined in a rotated coordinate system. The arguments of the rotation matrices $\mathfrak{D}_{M K}{ }^{L}$ are the Euler angles specifying the orientation of the rotated system. The phase convention used in this paper is that of Biedenharn and Rose. ${ }^{12}$ Then (7) becomes

$$
\begin{aligned}
K_{i f}=-(-)^{p-p^{\prime}} & \frac{Z^{2} e^{2}}{A M C^{2}} D^{1}-p,-p^{\prime} \delta_{i f}-\sum_{n} \sum_{L, L^{\prime}, M}(-)^{L^{\prime}-p^{\prime}} \\
& \times\left[C_{1 n}{ }^{L L^{\prime}}\left\langle f\left|\int \mathbf{j} \cdot \mathbf{B}_{L^{\prime} M^{k^{\prime} p^{\prime}}}\right| n\right\rangle\left\langle n\left|\int \mathbf{j} \cdot \mathbf{B}_{L p}{ }^{k p}\right| i\right\rangle+C_{2 n} L L^{\prime}\left\langle f\left|\int \mathbf{j} \cdot \mathbf{B}_{L p}{ }^{k p}\right| n\right\rangle\langle n| \int \mathbf{j} \cdot \mathbf{B}_{\left.L^{\prime} M^{k^{\prime} p^{\prime}}|i\rangle\right],}\right.
\end{aligned}
$$

where

$$
\begin{aligned}
C_{1 n} L L^{\prime} & =\frac{2 \pi}{c^{2}} i^{L+L^{\prime}}\left[(2 L+1)\left(2 L^{\prime}+1\right)\right]^{1 / 2} \frac{1}{E_{n}-E-i \Gamma_{n} / 2}, \\
C_{2 n} L L^{\prime} & =\frac{2 \pi}{c^{2}} i^{L+L^{\prime}}\left[(2 L+1)\left(2 L^{\prime}+1\right)\right]^{1 / 2} \frac{1}{E_{n}+E^{\prime}+i \Gamma_{n} / 2} .
\end{aligned}
$$

In the following we restrict ourselves to electric-dipole and -quadrupole radiation. We now rewrite Eq. (9) in terms of the nuclear polarizabilities $\mathfrak{P}_{L^{\prime}}{ }^{L}$, which are implicitly defined by the equation

$$
K_{i f}=(-)^{p^{\prime}} \sum_{L=1}^{2} \sum_{L^{\prime}=0}^{2 L}(-)^{I_{f}-M_{i}}\left(2 L^{\prime}+1\right)\left(\begin{array}{ccc}
L & L & L^{\prime} \\
p & M & -M-p
\end{array}\right)\left(\begin{array}{ccc}
L^{\prime} & I_{f} & I_{i} \\
M+p & -M_{f} & M_{i}
\end{array}\right) \Re_{L^{\prime}}{ }^{L} \mathscr{D}_{M,-p^{\prime}}(L) .
$$

It will be shown in the Appendix that this definition leads to the following explicit expression for the polarizability tensor:

$$
\mathfrak{B}_{L^{\prime}}{ }^{L}=(-)^{L} \sum_{n}\left\{\begin{array}{lll}
L^{\prime} & I_{f} & I_{i} \\
I_{n} & L & L
\end{array}\right\}\left[\mathfrak{夭}_{2 n}{ }^{L}+(-)^{L^{\prime}} \mathfrak{G}_{1 n}{ }^{L}\right]+\delta_{i f} \delta_{L 1} \delta_{L^{\prime} 0}(-)^{2 I_{i}}\left[3\left(2 I_{i}+1\right)\right]^{1 / 2} \frac{Z^{2} e^{2}}{A M c^{2}}
$$

where

$$
\begin{aligned}
& \left(\mathfrak{\mho}_{1 n}{ }^{L}=C_{1 n}{ }^{L L}\left\langle I_{f} \alpha_{f}\left\|\int \mathbf{j} \cdot \mathbf{A}_{L^{k^{\prime}}}(e)\right\| I_{n} \alpha_{n}\right\rangle\left\langle I_{n} \alpha_{n}\left\|\int \mathbf{j} \cdot \mathbf{A}_{L^{k}}(e)\right\| I_{i} \alpha_{i}\right\rangle,\right. \\
& \mathfrak{E}_{2 n}{ }^{L}=C_{2 n}{ }^{L L}\left\langle I_{f} \alpha_{f}\left\|\int \mathbf{j} \cdot \mathbf{A}_{L^{k}}(e)\right\| I_{n} \alpha_{n}\right\rangle\left\langle I_{n} \alpha_{n}\left\|\int \mathbf{j} \cdot \mathbf{A}_{L^{k^{\prime}}}(e)\right\| I_{i} \alpha_{i}\right\rangle .
\end{aligned}
$$

The reduced matrix elements of the Wigner-Eckart theorem are defined by

$$
\left\langle I_{f} M_{f}\left|T_{L M}\right| I_{i} M_{i}\right\rangle=(-)^{I_{f}-M_{f}}\left(\begin{array}{ccc}
I_{f} & L & I_{i} \\
-M_{f} & M & M_{i}
\end{array}\right)\left\langle I_{f}\left\|T_{L}\right\| I_{i}\right\rangle
$$

To compute the scattering cross section we need the absolute square of $K_{i f}$. Using

$$
\mathscr{D}_{M,-p^{\prime}}{ }^{L}(0, \theta, 0) D_{M,-p^{\prime}} K^{*}(0, \theta, 0)=(-)^{M+p^{\prime}} \sum_{J}(2 J+1)\left(\begin{array}{ccc}
L & K & J \\
M & -M & 0
\end{array}\right)\left(\begin{array}{ccc}
L & K & J \\
-p^{\prime} & p^{\prime} & 0
\end{array}\right) P_{J}(\cos \theta),
$$

${ }^{11}$ M. E. Rose, Elementary Theory of Angular Momentum (Interscience Publishers, Inc;, New York, 1957).

${ }^{12}$ L. C. Biedenharn and M. E. Rose, Rev. Mod. Phys. 25, 729 (1953). 
we obtain from (11)

$$
\begin{aligned}
\left|K_{i f}\right|^{2}= & \sum_{L, K=1}^{2} \sum_{J} \sum_{L^{\prime}=0}^{2 L} \sum_{K^{\prime}=0}^{2 K}\left(2 L^{\prime}+1\right)\left(2 K^{\prime}+1\right)(2 J+1)(-)^{M+p^{\prime}}\left(\begin{array}{ccc}
L & L & L^{\prime} \\
p & M & -M-p
\end{array}\right)\left(\begin{array}{ccc}
K & K & K^{\prime} \\
p & M & -M-p
\end{array}\right) \\
& \times\left(\begin{array}{cccc}
L^{\prime} & I_{f} & I_{i} \\
M+p & -M_{f} & M_{i}
\end{array}\right)\left(\begin{array}{ccc}
K^{\prime} & I_{f} & I_{i} \\
M+p & -M_{f} & M_{i}
\end{array}\right)\left(\begin{array}{ccc}
L & K & J \\
M & -M & 0
\end{array}\right)\left(\begin{array}{ccc}
L & K & J \\
-p^{\prime} & p^{\prime} & 0
\end{array}\right) \Re_{L^{\prime}} \mathscr{W}_{K_{K^{\prime}} K^{*} P_{J}(\cos \theta) .}
\end{aligned}
$$

This is the final formula which can be written before specifying the polarization of the beam and of the target nucleus. In the general case where the nuclei may be aligned or polarized one will have to use (16) directly, e.g., in the density-matrix formalism, ${ }^{13}$ to describe the experimental situation. We shall, however, at this time specialize to a nonaligned target. Then the cross sections are obtained by an incoherent averaging over the initial direction, i.e., summing over $M_{i}$. We also shall take the photons to be unpolarized. The cross section then is given by averaging over both orientation and polarization of the initial state of the nucleus and the photon, $M_{i}$ and $p$, respectively, and summing over the same quantities in the final state, $M_{f}$ and $p^{\prime}$. In this case we obtain

$$
\frac{d \sigma}{d \Omega}=\frac{1}{2 I_{i}+1} \frac{k^{\prime}}{k} \sum_{L, K=1}^{2} \sum_{L^{\prime}=0}^{2 L} g_{L^{\prime}} K L(\theta) \mathfrak{B}_{L^{\prime}}{ }^{L} \mathfrak{S}_{L^{\prime}}, K^{*},
$$

where

$$
\begin{aligned}
g_{L^{\prime}} K L(\theta) & \\
=(-) & \\
& \times\left(\begin{array}{ccc}
L & K & J \\
1 & -1 & 0
\end{array}\right)^{2}\left\{\begin{array}{lll}
L & K & J \\
K & L & L^{\prime}
\end{array}\right\} P_{J}(\cos \theta) .
\end{aligned}
$$

Obviously there holds

$$
g_{L}, K L(\theta)=g_{L^{\prime}} L R \text {. }
$$

The functions $g_{L^{\prime}}, K L(\theta)$ describe the different angular distributions. So $K=L=1$ describes the dipole photon scattering. $L^{\prime}=0$ is the scalar part, $L^{\prime}=1$ is the vector part, and $L^{\prime}=2$ is the tensor part. Similarly, $K=L=2$ describes the quadrupole scattering. $L^{\prime}$ here goes from 0 to 4 . The dipole-quadrupole interference is given by the terms $K=1, L=2$. These terms vanish at $\theta=90^{\circ}$. We have explicitly

$$
\begin{aligned}
& g_{0}{ }^{11}=\frac{1}{6}\left(1+\cos ^{2} \theta\right), \\
& g_{1}{ }^{11}=\frac{1}{4}\left(2+\sin ^{2} \theta\right), \\
& g_{2}{ }^{11}=\frac{1}{12}\left(13+\cos ^{2} \theta\right),
\end{aligned}
$$

${ }^{13}$ U. Fano, J. Opt. Soc. Am. 39, 859 (1949); Phys. Rev. 90, 577 (1953).

$$
\begin{aligned}
& g_{0}^{22}=\frac{1}{10}\left(1-3 \cos ^{2} \theta+4 \cos ^{4} \theta\right), \\
& g_{1}{ }^{22}=(1 / 20)\left(3+15 \cos ^{2} \theta-16 \cos ^{4} \theta\right), \\
& g_{2} 2^{22}=(1 / 28)\left(13-15 \cos ^{2} \theta+16 \cos ^{4} \theta\right), \\
& g_{3}^{22}=\frac{1}{5}\left(3-\cos ^{4} \theta\right), \\
& g_{4}^{22}=(1 / 35)\left(24+3 \cos ^{2} \theta+\cos ^{4} \theta\right), \\
& g_{0}{ }^{12}=-\left(1 / 15^{1 / 2}\right) \cos ^{3} \theta, \\
& g_{1}{ }^{12}=-\left(1 / 20^{1 / 2}\right) \cos \theta\left(3-2 \cos ^{2} \theta\right), \\
& g_{2}{ }^{12}=-\left(1 / 84^{1 / 2}\right) \cos \theta\left(3+2 \cos ^{2} \theta\right) .
\end{aligned}
$$

We now turn to the multipole matrix elements. By virtue of Siegert's theorem we have

where

$$
\int \mathbf{j} \cdot \mathbf{A}_{L M}(e) d \tau=-e \int \dot{\rho} \Phi_{L M} d \tau,
$$

$$
\Phi_{L M}=-\frac{1}{k}\left(\frac{L+1}{L}\right)^{1 / 2} \frac{(k r)^{L}}{(2 L+1) ! !} Y_{L M} .
$$

Introducing the multipole moment

$$
Q_{L M}=\int \rho r^{L} Y_{L M} d \tau
$$

we finally have

$$
\int \mathbf{j} \cdot \mathbf{A}_{L M}(e) d \tau=-e k^{L-1}\left(\frac{L+1}{L}\right)^{1 / 2} \frac{1}{(2 L+1) ! !} \dot{Q}_{L M} .
$$

The time derivative in (25) is simply

$$
\dot{Q}_{L M}=i \frac{E}{\hbar} Q_{L M} \text {. }
$$

We note that $Q_{L M}$ in (25) is normalized differently than usually in that $Y_{L M}$ is used instead of the usual Legendre polynomial $P_{L M}$.

Using Eqs. (26) and (27), Eq. (12) becomes

$$
\begin{aligned}
& \mathfrak{P}_{L^{\prime}}=\frac{4 \pi}{3(\hbar c)^{2}} \sum_{n}\left\{\begin{array}{ccc}
L^{\prime} & I_{f} & I_{i} \\
I_{n} & 1 & 1
\end{array}\right\} E E^{\prime} \\
& \times \times\left\langle I_{f}\left\|Q_{1}\right\| I_{n}\right\rangle\left\langle I_{n}\left\|Q_{1}\right\| I_{i}\right\rangle \\
& \times\left[\frac{(-)^{L^{\prime}}}{E_{n}-E-\frac{1}{2} i \Gamma_{n}}+\frac{1}{E_{n}+E^{\prime}+\frac{1}{2} i \Gamma_{n}}\right] \\
&+\delta_{i f} \delta_{L^{\prime} 0}(-)^{2 I_{i}}\left[3\left(2 I_{i}+1\right)\right]^{1 / 2} \frac{Z^{2} e^{2}}{A M c^{2}}
\end{aligned}
$$


and

$$
\begin{aligned}
\mathfrak{P}_{L^{2}}=\frac{\pi}{15} \frac{\left(E E^{\prime}\right)^{2}}{(\hbar c)^{4}} \sum_{n}\left\{\begin{array}{ccc}
L^{\prime} & I_{f} & I_{i} \\
I_{n} & 2 & 2
\end{array}\right\} \\
\times\left\langle I_{f}\left\|Q_{2}\right\| I_{n}\right\rangle\left\langle I_{n}\left\|Q_{2}\right\| I_{i}\right\rangle \\
\quad \times\left[\frac{(-)^{L^{\prime}}}{E_{n}-E-\frac{1}{2} i \Gamma_{n}}+\frac{1}{E_{n}+E^{\prime}+\frac{1}{2} i \Gamma_{n}}\right] .
\end{aligned}
$$

The absorption cross section then is given by

$$
\begin{aligned}
\sigma_{\mathrm{abs}} & =4 \pi \times \sum_{p, M_{i}} \frac{1}{2\left(2 I_{i}+1\right)} \operatorname{Im} K_{i i}\left(\theta=0, E=E^{\prime}\right) \\
& =\sigma_{\mathrm{abs}}{ }^{D}+\sigma_{\mathrm{abs}}{ }^{Q} .
\end{aligned}
$$

Here the dipole and the quadrupole cross sections are

$$
\sigma_{\mathrm{abs}}{ }^{D}=\frac{1}{2 I_{i}+1} \frac{32 \pi^{2}}{3} \frac{e^{2}}{\hbar c} \sum_{n} \frac{E^{2} \Gamma_{n} E_{n}\left|\left\langle n\left\|Q_{1}\right\| i\right\rangle\right|^{2}}{\left(E_{n}^{2}-E^{2}+\frac{1}{4} \Gamma_{n}^{2}\right)^{2}+E^{2} \Gamma_{n}^{2}}
$$

and

$$
\begin{aligned}
\sigma_{\mathrm{abs}}{ }^{Q}=\frac{1}{2 I_{i}+1} \frac{8 \pi^{2}}{75} & \frac{e^{2}}{(\hbar c)^{3}} \\
& \times \sum_{n} \frac{E^{4} \Gamma_{n} E_{n}\left|\left\langle n\left\|Q_{2}\right\| i\right\rangle\right|^{2}}{\left(E_{n}^{2}-E^{2}+\frac{1}{4} \Gamma_{n}^{2}\right)^{2}+E^{2} \Gamma_{n}^{2}},
\end{aligned}
$$

respectively.

Writing for the scattering cross section

$$
\frac{d \sigma}{d \Omega}=\frac{d \sigma_{D}}{d \Omega}+\frac{d \sigma_{Q}}{d \Omega}+\frac{d \sigma_{D Q}}{d \Omega},
$$

one finally obtains explicit expressions for the partial cross sections by inserting (28) and (29) in (17).

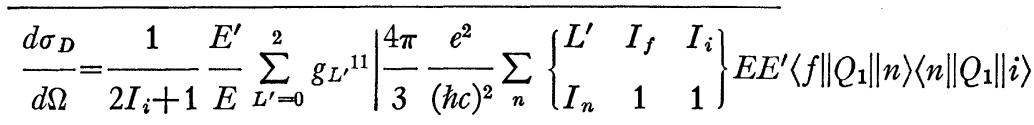

$$
\begin{aligned}
& \times\left[\frac{(-)^{L^{\prime}}}{E_{n}-E-\frac{1}{2} i \Gamma_{n}}+\frac{1}{E_{n}+E^{\prime}+\frac{1}{2} i \Gamma_{n}}\right]+\left.\delta_{i f} \delta_{L^{\prime} 0}(-)^{2 I_{i}}\left[3\left(2 I_{i}+1\right)\right]^{1 / 2} \frac{Z^{2} e^{2}}{A M c^{2}}\right|^{2}, \\
& \frac{d \sigma_{Q}}{d \Omega}=\frac{1}{2 I_{i}+1} \frac{E^{\prime}}{E} \sum_{L^{\prime}=0}^{4} g_{L^{2}}{ }^{22}\left|\frac{\pi e^{2}}{15} \frac{\left(E E^{\prime}\right)^{2}}{(\hbar c)^{4}} \sum_{n}\left\{\begin{array}{ccc}
L^{\prime} & I_{f} & I_{i} \\
I_{n} & 2 & 2
\end{array}\right\}\left\langle f\left\|Q_{2}\right\| n\right\rangle\left\langle n\left\|Q_{2}\right\| i\right\rangle\left[\frac{(-)^{L^{\prime}}}{E_{n}-E-\frac{1}{2} i \Gamma_{n}}+\frac{1}{E_{n}+E^{\prime}+\frac{1}{2} i \Gamma_{n}}\right]\right|^{2}, \\
& \frac{d \sigma_{D Q}}{d \Omega}=\frac{2}{2 I_{i}+1} \frac{E^{\prime}}{E} \sum_{L^{\prime}=0}^{2} g_{L^{\prime}}{ }^{12} \operatorname{Re}\left\{\left(\frac{4 \pi}{3} \frac{e^{2}}{\hbar c^{2}} \sum_{n}\left\{\begin{array}{ccc}
L^{\prime} & I_{f} & I_{i} \\
I_{n} & 1 & 1
\end{array}\right\} E E^{\prime}\left\langle f\left\|Q_{1}\right\| n\right\rangle\left\langle n\left\|Q_{1}\right\| i\right\rangle\right.\right.
\end{aligned}
$$

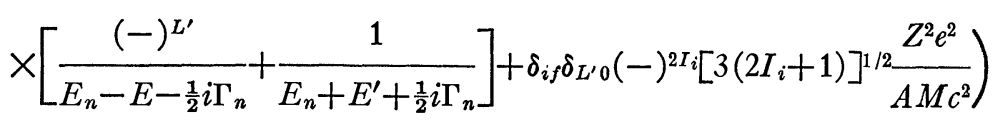

$$
\begin{aligned}
& \left.\times\left(\frac{\pi e^{2}}{15} \frac{\left(E E^{\prime}\right)^{2}}{(\hbar c)^{4}} \sum_{n}\left\{\begin{array}{ccc}
L^{\prime} & I_{f} & I_{i} \\
I_{n} & 2 & 2
\end{array}\right\}\left\langle f\left\|Q_{2}\right\| n\right\rangle\left\langle n\left\|Q_{2}\right\| i\right\rangle\left[\frac{(-)^{L^{\prime}}}{E_{n}-E-\frac{1}{2} i \Gamma_{n}}+\frac{1}{E_{n}+E^{\prime}+\frac{1}{2} i \Gamma_{n}}\right]\right)^{*}\right\} .
\end{aligned}
$$

In the special case $I_{i}=0$ (even-even nuclei) only the term $L^{\prime}=I_{f}$ contributes. Here also only the scalar part occurs in the elastic scattering.

\section{RESUME OF THE COLLECTIVE MODEL}

In this section we give a short review of the dynamic collective model of the giant resonance which has been developed in a series of earlier papers. ${ }^{3-6}$

The Hamiltonian for the collective surface degrees of freedom, the collective internal degrees of freedom, for the odd particle, and the various interactions between these degrees of freedom is

$$
\begin{aligned}
H= & H_{\mathrm{rot}}+H_{\mathrm{vib}}+H_{\mathrm{part}}+H_{\mathrm{dip}}+H_{\mathrm{quad}}+H_{\mathrm{dipvib}}+H_{\mathrm{quadvib}} \\
=\frac{\hbar^{2}}{2 J_{0}}\left(I^{2}-I_{3}{ }^{2}-\right. & \left.j_{3}{ }^{2}-d_{3}{ }^{2}-q_{3}{ }^{2}\right)+\frac{\hbar^{2}}{16 B \eta^{2}}\left[\left(I_{3}-j_{3}\right)^{2}+d_{3}{ }^{2}+q_{3}{ }^{2}-1\right]-\frac{\hbar^{2}}{8 B \eta^{2}}\left(I_{3}-j_{3}\right)\left(d_{3}+q_{3}\right) \\
& -\frac{\hbar^{2}}{2 B}\left(\frac{\partial^{2}}{\partial \xi^{2}}+\frac{1}{2} \frac{\partial^{2}}{\partial \eta^{2}}\right)+\frac{1}{2} C_{0} \xi^{2}+C_{2} \eta^{2}+H_{\mathrm{part}}+\sum_{\mu} \hbar \omega_{\mu}^{(1)}\left[1+G_{\mu}{ }^{(1)}(\xi+\mu \eta \sqrt{ } 6)\right] b_{\mu}{ }^{(1) \dagger} b_{\mu}{ }^{(1)} \\
& +\sum_{\nu} \hbar \omega_{\nu}^{(2)}\left[1+G_{\nu}{ }^{(2)}\left(\xi-\nu \delta_{1|\nu| \eta} \sqrt{ } 6\right)\right] b_{\nu}{ }^{(2) \dagger} b_{\nu}{ }^{(2)}+\hbar \omega^{(2)}(0)(0.284 / \sqrt{2})\left(b_{0}{ }^{(2) \dagger} b_{2}{ }^{(2)}+b_{2}{ }^{(2) \dagger} b_{0}{ }^{(2)}\right) \eta,
\end{aligned}
$$


with

$$
\begin{aligned}
\hbar \omega^{(1)} & =\hbar \omega^{(1)}(0) \frac{1+0.08(-2)^{-|\mu|} \tilde{\beta}_{0}}{1+(-2)^{-|\mu|} \tilde{\beta}_{0}}, \\
\hbar \omega_{\mu}^{(2)} & =\hbar \omega^{(2)}(0)\left[1+\left(\frac{1}{2} \mu^{2}-1\right) 0.284 \beta_{0}\right], \quad \hbar \omega^{(2)}(0)=(3.34 / 2.08) \hbar \omega^{(1)}(0), \\
G_{\mu}^{(1)} & =(-2)^{-|\mu|}(5 / 4 \pi)^{1 / 2}\left(-\frac{1}{1+(-2)^{-|\mu|} \tilde{\beta}_{0}}+\frac{0.08}{1+0.08(-2)^{-|\mu|} \tilde{\beta}_{0}}\right) \\
G_{\nu}^{(2)} & =\frac{\left(\frac{1}{2} \nu^{2}-1\right) 0.284 \beta_{0}}{1+\left(\frac{1}{2} \nu^{2}-1\right) 0.284 \beta_{0}}, \\
\tilde{\beta}_{0} & =(5 / 4 \pi)^{1 / 2} \beta_{0} .
\end{aligned}
$$

$b^{(1)}$ and $b^{(2)}$ are the annihilation operators for the dipole and quadrupole giant resonances, respectively. For $H_{\text {part }}$ we use the Nilsson Hamiltonian

$$
H_{\mathrm{part}}=\frac{1}{2} F_{w}\left(-\nabla^{2}+K^{2} r^{2}\right)-F_{w} \beta_{0} r^{2} Y_{20}+C_{w} \mathbf{l} \cdot \mathbf{s}+C_{w} \mathbf{1}^{\prime 2} .
$$

The refinements included in this paper which go beyond the treatment of the earlier work are the following. Firstly, the differential equation for the $\eta$ vibrations has been solved numerically since the accuracy of the perturbation treatment used previously was not sufficient. Secondly, the off-diagonal term

$$
\left(\hbar^{2} / 8 B \eta^{2}\right)\left(I_{3}-j_{3}\right)\left(d_{3}+q_{3}\right)
$$

has been taken into account. ${ }^{14}$ The first change resulted in a slight increase in the energy spacing of the upper states and in a substantial change in the absorption strengths of the vibrational satellites. The second change increases the spacing between the main upper lines by about $150 \mathrm{keV}$.

The wave functions for the Hamiltonian (36), excluding the off-diagonal term (39), are

$$
\begin{aligned}
& \left|I M K, \Omega \alpha, n_{2} n_{0}, m s, n t\right\rangle=\left(\frac{2 I+1}{32 \pi^{2}}\right)^{1 / 2} u_{n_{0}, m s, n t}(\xi)\left\{\left[\mathscr{D}_{M K} I \chi_{\Omega \alpha}-(-)^{I+j+K+\Omega+\frac{1}{2} s(s+1)+t}\left(\operatorname{sgn} t+\delta_{t, 0}\right) \mathscr{D}_{M,-K} \chi_{-\Omega \alpha}\right]\right. \\
& \times \psi_{m s}{ }^{(1)} \psi_{n t}{ }^{(2)} \varphi_{|K-\Omega| n_{2}, m s, n t}(-\eta)+(-)^{\frac{1}{2}(K-\Omega-s(s-1)-|t|}\left[\mathscr{D}_{M K}{ }^{I} \chi_{\Omega \alpha}-(-)^{I+j+K+\Omega+\frac{1}{2} s(s-1)}\left(\operatorname{sgn} t+\delta_{t, 0}\right) D_{M,-K}{ }^{I} \chi_{-\Omega \alpha}\right] \\
& \left.\times \psi_{m-s}{ }^{(1)} \psi_{n(-)^{t}{ }_{t}^{(2)}} \varphi_{|K-\Omega| n_{2}, m s, n t}(\eta)\right\},
\end{aligned}
$$

where

$$
\operatorname{sgn} \mu=\mu /|\mu| \text { for } \mu \neq 0, \quad \operatorname{sgn} 0=0 .
$$

The meaning of the quantum numbers is the following: $I, M$, and $K$ are the total spin and its projection on the laboratory and intrinsic axis, respectively; $\Omega$ and $\alpha$ are the quantum numbers of the Nilsson state; $n_{2}$ and $n_{0}$ describe the $\eta$ and $\xi$ vibrations, respectively; $m$ and $n$ give the number of dipole and quadrupole giant resonance phonons, respectively, and $s$ and $t$ are their Cartesian classifications. Since a Nilsson wave function does not have a good angular momentum, the symbol $(-)^{j}$ is to be considered as an operator. The off-diagonal interaction (39) mixes the Cartesian giant resonance components $s$ and $-s$, and separately $t$ and $-t$.

The symmetries contained in (40) impose the following conditions upon the states.

For $m=1, n=0$ we have ${ }^{15}$

$$
K=\Omega-|S|, \Omega-|S|+2, \Omega-|S|+4, \cdots, \quad I=|K|,|K|+1,|K|+2, \cdots,
$$

and for $m=0, n=1$ we have

$$
K=\Omega-|t|, \Omega-|t|+2, \Omega-|t|+4, \cdots, \quad I=|K|,|K|+1,|K|+2, \cdots .
$$

The energies corresponding to the wave functions (40) are

$$
\begin{aligned}
E_{I K, \Omega \alpha, m s, n t, n_{2}, n_{0}}=[I(I+1)- & \left.K^{2}-s^{2}-t^{2}-\Omega^{2}\right] E_{R}+\left(n_{0}+\frac{1}{2}\right) E_{\beta}-m\left(\hbar \omega_{s}{ }^{(1)} G_{s}{ }^{(1)} / E_{\beta}\right)^{2} \\
& \times 3 E_{R} \beta_{0}{ }^{2}-n\left(\hbar \omega_{t}{ }^{(2)} G_{t}{ }^{(2)} / E_{\beta}\right)^{2} 3 E_{R} \beta_{0}{ }^{2}+m \hbar \omega_{s}{ }^{(1)}+n \hbar \omega_{t}{ }^{(2)}+\epsilon_{\Omega \alpha}+E_{|K-\Omega|, n_{2}, m s, n t} .
\end{aligned}
$$

${ }^{14}$ This term has also been considered by S. F. Semenko, Phys. Letters 13, 157 (1964); Yadernaya Fiz. 1, 414 (1965) [English transl.: Soviet J. Nucl. Phys. 1, 295 (1965)]. We thank her for a private communication.

${ }_{15}$ The selection rule, as given in Ref. 4 , contains an error for $\Omega=\frac{1}{2}$. 
The last term in (43) gives the energy of the $\eta$ vibrations. For $s=0, t=0,2$ it can be given explicitly:

$$
E_{|K-\Omega| n_{2}, m 0, n t}=\left\{2 n_{2}+\frac{1}{2}\left[(K-\Omega)^{2}+t^{2}\right]^{1 / 2}\right\} E_{\gamma} .
$$

For the other cases this energy has been obtained by numerical solution of the equation for the $\eta$ vibrations.

As final step we now consider the off-diagonal operator (39). That means that we still have to diagonalize a two-by-two matrix which has diagonal elements given by (43) and off-diagonal elements which have to be computed numerically. They are

(1) For one giant dipole phonon,

$$
\begin{aligned}
\left\langle I^{\prime} K^{\prime}, \Omega^{\prime} \alpha^{\prime}, n_{2}^{\prime} n_{0}^{\prime}, 1 S^{\prime}, 00\right| & -\frac{3 E_{R} d_{3}\left(I_{3}-j_{3}\right)}{16 \eta^{2}} \beta_{0}^{2}\left|I K, \Omega \alpha, n_{2} n_{0}, 1 S, 00\right\rangle \\
& =-\delta_{I I^{\prime}} \delta_{K K^{\prime}} \delta_{\Omega \Omega^{\prime}} \delta_{\alpha \alpha^{\prime}} \delta_{n_{0} n_{0}} \delta_{S,-S^{\prime}} i(3 / 16) E_{R} \beta_{0}{ }^{2}(K-\Omega) S\left\langle\varphi_{|K-\Omega| n_{n^{\prime}} 1 S^{\prime} 00}\left|\eta^{-2}\right| \varphi_{|K-\Omega| n_{2} 1 S 00}\right\rangle .
\end{aligned}
$$

(2) For one giant quadrupole phonon,

$$
\begin{aligned}
\left\langle I^{\prime} K^{\prime}, \Omega^{\prime} \alpha^{\prime}, n_{2}{ }^{\prime} n_{0}{ }^{\prime}, 00,1 t\right. & -\frac{3 E_{R} \beta_{0}{ }^{2} 9_{3}\left(I_{3}-j_{3}\right)}{16 \eta^{2}}\left|I K, \Omega \alpha, n_{2} n_{0}, 00,1 t^{\prime}\right\rangle \\
& =-\delta_{I I^{\prime}} \delta_{K K^{\prime}} \delta_{\Omega \Omega^{\prime}} \delta_{\alpha \alpha^{\prime}} \delta_{n_{0} n_{0^{\prime}}} \delta_{t,-t^{\prime}}(3 / 16) E_{R} \beta_{0}{ }^{2}(K-\Omega)|t|\left\langle\varphi_{|K-\Omega| n_{2^{\prime}}, 00,1 t^{\prime}}\left|\eta^{-2}\right| \varphi_{|K-\Omega| n_{2}, 00,1 t}\right\rangle .
\end{aligned}
$$

This completes the computation of the energy spectrum.

\section{PHOTON-INTERACTION MATRIX ELEMENTS}

In the computation of the reduced matrix elements of the multipole operators (25) needed in (30) and (32), two steps can be distinguished: (i) The operators (25) have to be written in terms of the giant resonance and surface coordinates in the intrinsic system. (ii) The matrix elements of these operators between the various states have to be evaluated. After expressing the operators in the intrinsic system they can be expanded in terms of the surface parameters. We shall limit ourselves to terms quadratic in the static deformation parameter $\beta_{0}$ and to terms linear in the vibrational amplitudes $\xi$ and $\eta$. This leads to the following expressions for the intrinsic components of the dipole and quadrupole operator:

$Q_{1 \mu}=d_{|\mu|}{ }^{(1)}\left\{\left[i^{|\mu|}\left(b_{|\mu|}{ }^{(1) \dagger}+b_{|\mu|}{ }^{(1)}\right)-\mu\left(b_{-|\mu|}{ }^{(1)} \dagger+b_{-|\mu|}{ }^{(1)}\right)\right]\right.$ $\left.\times\left[1+S_{\mu}{ }^{(1)} \xi\right]+\left[i^{|\mu|}\left(b_{|\mu|}{ }^{(1)} \dagger+b_{|\mu|}{ }^{(1)}\right)+\mu\left(b_{-|\mu|}{ }^{(1)} \dagger+b_{-|\mu|}{ }^{(1)}\right)\right](|\mu| \sqrt{ } 6) S_{\mu}{ }^{(1)} \eta\right\}$,

with

and

$$
\begin{aligned}
d_{0}{ }^{(1)} & =\hbar\left(\frac{3}{8 \pi} \frac{N Z}{A} \frac{(1+\alpha)}{M} \frac{1}{\hbar \omega_{0}^{(1)}}\right)^{1 / 2}\left(0.925+0.33 \beta_{0}+0.323 \beta_{0}{ }^{2}\right), \\
d_{1}{ }^{(1)} & =\left(\frac{3}{8 \pi} \frac{N Z}{A} \frac{(1+\alpha)}{M} \frac{1}{\hbar \omega_{0}^{(1)}}\right)^{1 / 2}\left(-0.654+0.114 \beta_{0}-0.14 \beta_{0}{ }^{2}\right), \\
S_{\mu}{ }^{(1)} & =(-2)^{-|\mu|} 0.349
\end{aligned}
$$

$Q_{2 \mu}=d_{|\mu|}{ }^{(2)}\left\{\left[i^{\delta|\mu| 1}\left(b_{|\mu|}{ }^{(2) \dagger}+b_{|\mu|}{ }^{(2)}\right)-\operatorname{sgn} \mu i^{\delta|\mu| 2}\left(b_{-|\mu|}{ }^{(2) \dagger}+b_{-|\mu|}{ }^{(2)}\right)\right]\right.$

with

$$
\left.\times\left[1+S_{\mu}^{(2)} \xi\right]-\left[i^{\delta|\mu| 1}\left(b_{2-|\mu|}{ }^{(2) \dagger}+b_{2-|\mu|}{ }^{(2)}\right)+\operatorname{sgn} \mu\left(b_{|\mu|-2}{ }^{(2)} \dagger+b_{|\mu|-2}{ }^{(2)}\right)\right] \sqrt{2}\left(\frac{1}{2} \sqrt{3}\right)^{\delta|\mu| 1} 0.497 \eta\right\},
$$

$$
\begin{aligned}
& d_{0}^{(2)}=\hbar\left(\frac{6}{\pi} \frac{N Z}{A} \frac{(1+\alpha)}{M} \frac{1}{\hbar \omega_{0}^{(2)}}\right)^{1 / 2} R_{0} 0.311\left(1+0.497 \beta_{0}+1.21 \beta_{0}{ }^{2}\right), \\
& d_{1}^{(2)}=-\hbar\left(\frac{3}{\pi} \frac{N Z}{A} \frac{(1+\alpha)}{M} \frac{1}{\hbar \omega_{0}{ }^{(2)}}\right)^{1 / 2} R_{0} 0.311\left(1+0.248 \beta_{0}+0.42 \beta_{0}{ }^{2}\right), \\
& d_{2}{ }^{(2)}=\hbar\left(\frac{3}{\pi} \frac{N Z}{A} \frac{(1+\alpha)}{M} \frac{1}{\hbar \omega_{0}^{(2)}}\right)^{1 / 2} R_{0} 0.311\left(1-0+97 \beta_{0}+0.29 \beta_{0}{ }^{2}\right), \\
& S_{\mu}{ }^{(2)}=\frac{2-\mu^{2}}{2} 0.497 .
\end{aligned}
$$


The parameter $\alpha$ has been introduced originally to account for the exchange forces in terms of an effective nuclear mass. Here it is used strictly as a scaling factor to adjust the absolute magnitude of the cross sections in a given nucleus.

The evaluation of the matrix elements of these operators using the wave functions (40) is lengthy but straightforward. The final results are

(1) Even-even nuclei:

$$
\begin{aligned}
& \left\langle I^{\prime} K^{\prime}, n_{2}^{\prime} n_{0}^{\prime}, 1 S, 00\left\|Q_{1}\right\| I K, n_{2} n_{0}, 00,00\right\rangle=\left(\frac{\left(2 I^{\prime}+1\right)(2 I+1)}{8\left(1+\delta_{K 0}\right)\left(1+\delta_{K^{\prime} 0}\right)}\right)^{1 / 2} \sum_{\nu=-1}^{1}(-)^{I+K^{\prime}+1} d_{|\nu|}^{(1)}\left(i^{|\nu|} \delta_{S|\nu|}-\nu \delta_{S,-\mid \nu i}\right) \\
& \times\left[\pi_{\varphi}+(-)^{\left(\nu-K^{\prime}\right) / 2}\right]\left[1+(-)^{K^{\prime}+|S|}\right]\left[\left(\begin{array}{ccc}
I & 1 & I^{\prime} \\
K & \nu & -K^{\prime}
\end{array}\right)+(-)^{I}\left(\begin{array}{ccc}
I & 1 & I^{\prime} \\
-K & \nu & -K^{\prime}
\end{array}\right)\right] \\
& \times\left(\left\langle\varphi_{K^{\prime} n^{\prime} 1 S 00} \mid \varphi_{K n_{2} 0000}\right\rangle\left\langle u_{n_{0^{\prime}} 1 S 00}\left|1+S_{v}^{(1)} \xi\right| u_{n 00000}\right\rangle\right. \\
& \left.+(-)^{S(S+1) / 2}\left\langle\varphi_{K^{\prime} n_{2}{ }^{\prime} 1 S 00}\left|(|\nu| \sqrt{ } 6) S_{\nu}{ }^{(1)} \eta\right| \varphi_{K n_{2} 0000}\right\rangle\left\langle u_{n^{\prime} 1 S 00} \mid u_{n_{0} 0000}\right\rangle\right) .
\end{aligned}
$$

Here $\pi_{\varphi}$ is the parity of $\varphi_{K n_{2} 0000}$.

$$
\begin{aligned}
& \left\langle I^{\prime} K^{\prime}, n_{2}^{\prime} n_{0}^{\prime}, 00,1 t\left\|Q_{2}\right\| I K, n_{2} n_{0}, 0000\right\rangle \\
& =(-)^{I+K^{\prime}}\left(\frac{\left(2 I^{\prime}+1\right)(2 I+1)}{8\left(1+\delta_{K 0}\right)\left(1+\delta_{K^{\prime} 0}\right)}\right)^{1 / 2} \sum_{\nu=-2}^{2} d_{|\nu|}(2)\left[\left(\begin{array}{ccc}
I & 2 & I^{\prime} \\
K & \nu & -K^{\prime}
\end{array}\right)+(-)^{I}\left(\begin{array}{ccc}
I & 2 & I^{\prime} \\
-K & \nu & -K^{\prime}
\end{array}\right)\right], \\
& \times\left[1+\pi_{\varphi}(-)^{(1 / 2)\left[K^{\prime}-|t|+\nu(\nu+1)+|t|(|t|-1)\right]}\right]\left[A_{\nu}{ }^{t}+\left(\operatorname{sgn} t+\delta_{0 t}\right)(-)^{K^{\prime}+t} A_{-\nu}{ }^{t}\right] \\
& A_{\nu}{ }^{t}=(-)^{\nu}\left(\operatorname{sgn} t+\delta_{t 0}\right)\left(\left(\delta_{t|\nu|}-\operatorname{sgn} \nu \delta_{t,-|\nu|}\right)\left\langle u_{n 0^{\prime} 001 t}\left|1+S_{\nu}{ }^{(2)}\right| u_{n_{0000}}\right\rangle\left\langle\varphi_{K^{\prime} n_{2}, 001 t} \mid \varphi_{K n_{2} 0000}\right\rangle\right. \\
& \text { (2) Odd- } A \text { nuclei: } \\
& \left.-\left(\delta_{t, 2-|\nu|}+\operatorname{sgn} t \delta_{-t, 2-|\nu|}\right)\left\langle u_{n 0^{\prime} 001 t} \mid u_{n_{000000}}\right\rangle\left\langle\varphi_{K^{\prime} n_{2^{\prime}} 001 t}\left|\sqrt{2}(\sqrt{3} / 2)^{\delta_{|\nu| 1}} 0.497 \eta\right| \varphi_{K n_{2} 0000}\right\rangle\right) \text {. }
\end{aligned}
$$

$$
\begin{aligned}
& \left\langle I^{\prime} K^{\prime}, \Omega^{\prime} \alpha^{\prime}, n_{2}^{\prime} n_{0}^{\prime}, 1 S, 00\left\|Q_{1}\right\| I K, \Omega \alpha, n_{2} n_{0}, 00,00\right\rangle \\
& =(-)^{I+K^{\prime}+:} \delta_{\Omega^{\prime} \Omega} \delta_{\alpha^{\prime} \alpha}\left[(1 / 8)\left(2 I^{\prime}+1\right)(2 I+1)\right]^{1 / 2} \sum_{\nu=-1}^{1} d_{|\nu|}\left(i^{|\nu|} \delta_{S|\nu|}-\nu \delta_{S,-|\nu|}\right)\left(\begin{array}{ccc}
I & 1 & I^{\prime} \\
K & \nu & -K^{\prime}
\end{array}\right) \\
& \times\left[\pi_{\varphi}+(-)^{\frac{1}{2}\left(\nu-K^{\prime}+\Omega^{\prime}\right)}\right]\left[1+(-)^{K-K^{\prime}-|S|}\right]\left(\left\langle\varphi_{\left|K^{\prime}-\Omega\right| n_{2^{\prime}} 1 S 00} \mid \varphi_{|K-\Omega| n_{2} 0000}\right\rangle\left\langle u_{n_{0^{\prime}} 1 S 00}\left|1+S_{\nu}{ }^{(1)} \xi\right| u_{n_{0} 0000}\right\rangle\right. \\
& \left.+(-)^{S(S+1) / 2}\left\langle\varphi_{\left|K^{\prime}-\Omega\right| n^{\prime} 1 S 00}\left|(|\nu| \sqrt{ } 6) S_{\nu}{ }^{(1)} \eta\right| \varphi_{|K-\Omega| n_{2} 0000}\right\rangle\left\langle u_{n_{0}^{\prime} 1 S 00} \mid u_{n_{0} 0000}\right\rangle\right) \text {, } \\
& \left\langle I^{\prime} K^{\prime}, \Omega^{\prime} \alpha^{\prime}, n_{2}^{\prime} n_{0}^{\prime}, 00,1 t\left\|Q_{2}\right\| I K, \Omega \alpha, n_{2}, n_{0}, 00,00\right\rangle \\
& =(-)^{I-K^{\prime}} \delta_{\Omega \Omega^{\prime}} \delta_{\alpha \alpha^{\prime}}\left[(1 / 8)\left(2 I^{\prime}+1\right)(2 I+1)\right]^{1 / 2} \sum_{\nu=-2}^{2} d_{|\nu|}{ }^{(2)}\left(\begin{array}{ccc}
I & 2 & I^{\prime} \\
K & \nu & -K^{\prime}
\end{array}\right) \\
& \times\left[1+\pi_{\varphi}(-)^{\left[K^{\prime}-\Omega-|t|+\nu(\nu-1)+|t|(|t|-1)\right] / 2}\right]\left[A_{\nu}{ }^{t}+\left(\operatorname{sgn} t+\delta_{0 t}\right)(-)^{K^{\prime}-I+t} A_{-\nu}{ }^{t}\right], \\
& A_{\nu}{ }^{t}=(-)^{\nu}\left(\operatorname{sgn} t+\delta_{0 t}\right)\left[\left(\delta_{t \mid \nu} i^{\delta_{|\nu| 1}}-\operatorname{sgn} \nu i^{\delta / \nu \mid 2} \delta_{t,-|\nu|}\right)\left\langle u_{n 0^{\prime} 001 t}\left|1+S_{\nu}{ }^{(2)} \xi\right| u_{n_{0} 0000}\right\rangle\left\langle\varphi_{\left|K^{\prime}-\Omega\right| n_{2^{\prime}} 001 t} \mid \varphi_{|K-\Omega| n_{2} 0000}\right\rangle\right.
\end{aligned}
$$

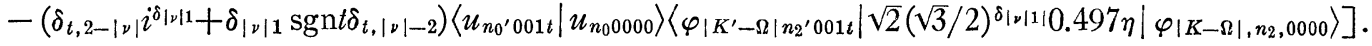

These matrix elements contain overlap integrals of the $\eta$-vibration wave functions, which have been evaluated numerically. ${ }^{16}$

\section{ANALYSIS OF EXPERIMENTAL DATA}

Up to now, scattering experiments have been performed only for four heavy deformed nuclei, namely,

${ }_{16}$ The matrix elements for the dipole operator in odd- $A$ nuclei as given in Ref. 4 contain an error in the phases. As a result of this error the $\eta$-parity selection rule discussed in that paper is wrong. We acknowledge discussions with E. G. Fuller concerning this point.
$\mathrm{Tb}, \mathrm{Ho}, \mathrm{Er}, \mathrm{Ta}$. We shall try to give as complete as possible an analysis for these four cases.

The analysis proceeds in the following manner. Among the parameters determining the theoretical cross sections the three parameters $E_{R}, E_{\gamma}$, and $E_{\beta}$ are taken from the low-energy spectra. The deformation parameter $\beta_{0}$ can, in principle, also be determined from the low-energy data. We still consider $\beta_{0}$ to be an adjustable parameter, both because it is not too well determined by the Coulomb-excitation experiment, and in order to check for over-all consistency between the 
values of $\beta_{0}$ obtained by both methods. The other parameters determine, crudely, the position, absolute magnitude, and widths of the resonances. For the width of the different dipole resonances we assume that they only depend on the excitation energy and we describe this by a power law, i.e., we put

$$
\Gamma_{n}=\Gamma_{0}\left(E_{n} / E_{0}\right)^{\delta} .
$$

All widths thus are described by two parameters $\Gamma_{0}$ and $\delta$. Finally, we are left with two parameters, namely, $E_{0}$, the position of the low-energy giant resonance, and $\alpha$, the exchange correction to the integrated cross section $^{17,18}$ (effective-mass correction of the hydrodynamic model).

The adjustment of the five parameters would be an almost insurmountable job. Fortunately in this case, different features of the cross section have different sensitivity with respect to the different parameters. Thus the parameters $E_{0}, \Gamma_{0}$, and $\alpha$ are practically fixed by the low-energy peak of the absorption cross section. The remaining two parameters $\beta_{0}$ and $\delta$ are then determined by the over-all splitting and by the height of the upper bump, respectively. The fitting thus consists in an iterative procedure going through the above sequence of parameter adjustments until a satisfactory fit has been obtained.

In odd- $A$ nuclei the low-energy parameters are not necessarily available from the low-energy data. In such cases parameters from neighboring nuclei were used. This procedure evidently introduces some uncertainties. This is particularly true for the vibrational energy $E_{\gamma}$. In these cases, therefore, $E_{\gamma}$ was also varied while fitting the theoretical curve to the experimental data.

The precision with which the different parameters could be determined was highest for $E_{0}$ (less than $1 \%$ ), $\Gamma_{0}$ (about $5 \%$ ), and $\beta_{0}$ (about $10 \%$ ). It should be noted that the different parameters can be slightly changed by making small changes in other parameters. A correct determination of the region of best fit would have required extensive numerical computations. These were, however, not carried out. The above-quoted uncertainties include an estimated uncertainty resulting from this interrelation of the parameters. Unfortunately, the accuracy in the absolute magnitude of the experimental cross sections still seems to preclude a complete intercomparison between absorption and scattering data. The conclusions of our paper are thus based mostly on fits to the energy dependence of the different cross sections, and no definite conclusions can be drawn concerning the parameter $\alpha$, i.e., on the magnitude of the integrated cross section.

We now proceed to the discussion of the different nuclei.

${ }^{17}$ J. S. Levinger and H. A. Bethe, Phys. Rev. 78, 115 (1950). ${ }_{18}$ M. Gell-Mann, M. L. Goldberger, and W. E. Thirring, Phys. Rev. 95, 1612 (1954).
HEA VY DEFORMED N UCLEI

TABLE I. Resonance parameters for erbium.

\begin{tabular}{cccccccc}
\hline $\begin{array}{c}E_{R} \\
(\mathrm{keV})\end{array}$ & $\begin{array}{c}E_{\gamma} \\
(\mathrm{keV})\end{array}$ & $\begin{array}{c}E_{\beta} \\
(\mathrm{MeV})\end{array}$ & $\begin{array}{c}E_{0} \\
(\mathrm{MeV})\end{array}$ & $\begin{array}{c}\Gamma_{0} \\
(\mathrm{MeV})\end{array}$ & $\beta_{0}$ & $\delta$ & $\alpha$ \\
\hline 11.68 & 758 & 1.46 & 12.1 & 2.2 & 0.29 & 1.5 & 0.09 \\
\hline \hline
\end{tabular}

\section{Erbium}

The photon-absorption experiment ${ }^{19}$ has been performed only on natural erbium in which the abundances of the different even-even isotopes are $33.4 \%$ for ${ }^{166} \mathrm{Er}$, $27.1 \%$ for ${ }^{168} \mathrm{Er}$, and $14.9 \%$ for ${ }^{170} \mathrm{Er}$. The low-energy spectra are well known for ${ }^{166} \mathrm{Er}$ and ${ }^{168} \mathrm{Er}$, not so well known for ${ }^{170} \mathrm{Er}$. The low-energy parameters are practically the same for ${ }^{166} \mathrm{Er}$ and ${ }^{168} \mathrm{Er}$. The computations thus were performed with the parameters of the most abundant isotope ${ }^{166} \mathrm{Er}$. The fit obtained is shown in Fig. 1. The parameters are given in Table I. ${ }^{20}$

The level scheme and the dipole strengths for the giant resonances are shown in Fig. 2. The dashed line shows the position and the strength of the transversal mode if the coupling to the surface vibrations is omitted (Danos-Okamoto picture). The main effect of the coupling to the surface mode is a splitting of the transversal mode by almost $2 \mathrm{MeV}$. Many vibrational satellites also appear. However, only one of them acquires an appreciable dipole strength.

The scattering cross sections, which result with the above obtained parameters, are shown in Figs. 3 to 5 . Both the elastic and the Raman scattering cross sections are given. The largest cross sections are shown in Fig. 3. The elastic scattering here is purely scalar, since the ground-state spin vanishes. Tensor scattering is included in Fig. 3, leading to the first rotational state

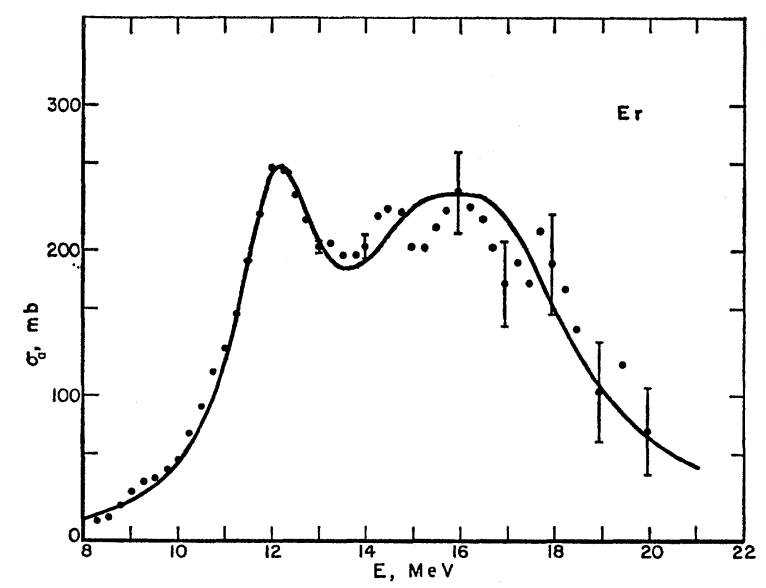

Fig. 1. $\gamma$-absorption cross section of Er, experimental data from Refs. 19 and 20.

${ }^{19}$ E. G. Fuller and E. Hayward, Nucl. Phys. 30, 613 (1962)

${ }^{20}$ As a result of a redetermination of the neutron-detector efficiency, the cross sections in Refs. 19 and 28 have to be multiplied by 0.67 and 0.75 , respectively [E. G. Fuller and H. Gerstenberg (private communication)]. 


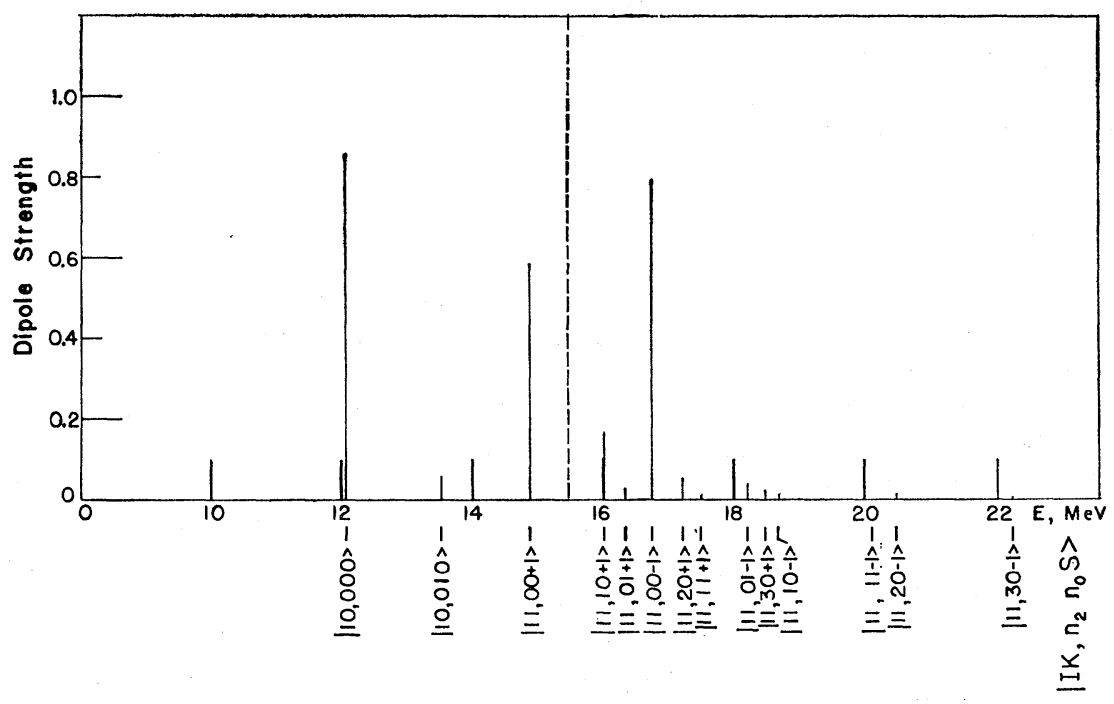

Fig. 2. Level scheme and dipole strengths of the giant dipole resonance states of Er. The dashed line shows position and strength of the transverse mode for an axially symmetric deformation.

$(2+)$ and to the $\gamma$-band head. The latter cross section is remarkable. It is by about an order of magnitude larger than the vibrational Raman scattering cross sections shown in Figs. 4 and 5. The reason for this is the following. The scattering strengths [see Eq. (48)] contain overlap integrals between the $\eta$-vibrational wave functions. They are in general very small and would even vanish exactly except for the presence of the rotation-vibration and dipole-vibration interaction. Explicitly, this effect results from the presence of the term $\left(I_{3}{ }^{2}+d_{3}{ }^{2}\right) /\left(16 B \eta^{2}\right)$, Eq. (36). Thus the differential equation for the wave function of the $\eta$ vibrations is different for different bands. However, the overlap integrals are still small for those wave functions which would have been orthogonal in the absence of the above term. The structure of the $\eta$-vibration band head, as seen from its quantum numbers $I=2, K=2, n_{2}=0$, and $n_{0}=0$, actually involves no $\eta$-vibration phonon. It merely indicates a kind of centrifugal stretching resulting from the finite $K$, which leads to a dynamic perma-

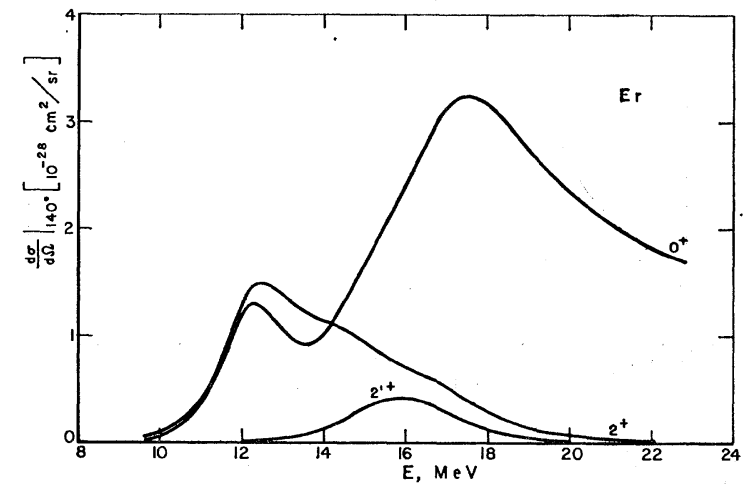

FIG. 3. Calculated elastic and inelastic scattering cross sections of Er for scattering into the ground-state band $\left(0^{+}, 2^{+}\right)$and the $\gamma$ band $\left(2^{\prime+}\right)$. nent triaxially deformed shape. The energy of this state is connected with the genuine $\eta$-vibration energy via the restoring forces. The absence of a vibrational kinetic energy also shows up in Eq. (44). An energy $2 E_{\gamma}$ is associated with a genuine vibration while these pseudovibrations are associated with energies $E_{\gamma}$.

The quadrupole scattering associated with the E2 giant resonances has also been computed. It is not separately shown, since its magnitude is generally small. However, it shows up in interference effects with dipole scattering. The expected angular distributions are plotted for several energies in Fig. 6. The pure dipole distributions are indicated by dashed lines. The only experimental data ${ }^{21}$ available at this time are also plotted. One cannot claim agreement between theory and experiment at $20 \mathrm{MeV}$, where the interference

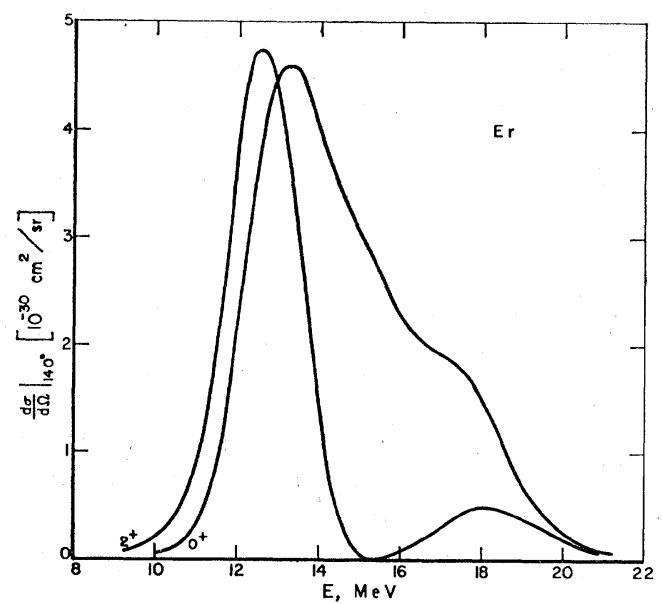

FIG. 4. Calculated inelastic scattering cross sections of $\mathrm{Er}$ for scattering into the $\beta$ band.

${ }^{21}$ M. Langevin, J. M. Loiscaux, and J. M. Maison, Nucl. Phys. 54, 114 (1964). 
effects are largest. ${ }^{22}$ However, the agreement is satisfactory at lower energies.

The total quasi-elastic scattering cross section at $140^{\circ}$ is shown in Fig. 7. The experimental points are from Ref. 21. The agreement is excellent, bearing in mind that no adjustments have been made in the parameters obtained from a fit to the absorption data.

\section{Holmium}

As compared with erbium, the situation in holmium is, on the one hand, clearer because the nucleus is monoisotopic; on the other hand, it is more uncertain because no consistent analysis of the low-energy data is available as yet. However, two $\gamma$-band heads with spins $\frac{3}{2}-$ and $11 / 2^{-}$at 514 and $687 \mathrm{keV},{ }^{23}$ respectively, seem to be

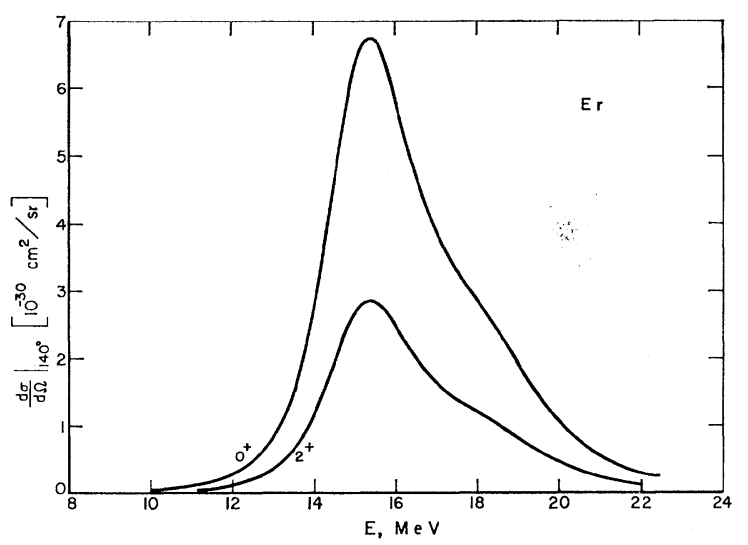

FIG. 5. Calculated inelastic scattering cross sections of Er for scattering into the higher $\gamma$ band.

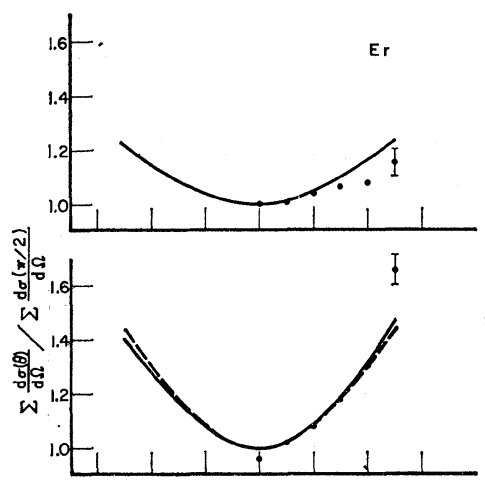

FIG. 6. Angular distributions of the total quasi-elastic scattering cross section of Er for different energies. The dashed line shows the angular distribution for pure dipole scattering. The experimental points are taken from Ref. 21.

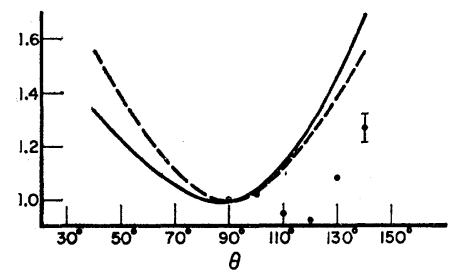

Top: $11.5-14 \mathrm{MeV}$ middle: $\quad 14-17.3$ $\mathrm{MeV}$, bottom: 17.5 $20 \mathrm{MeV}$.

${ }^{22}$ A similar observation has already been made by E. G. Fuller (private communication).

${ }_{23}$ R. M. Diamond, B. Elbek, and F. S. Stephens, Nucl. Phys. 43, 560 (1963).

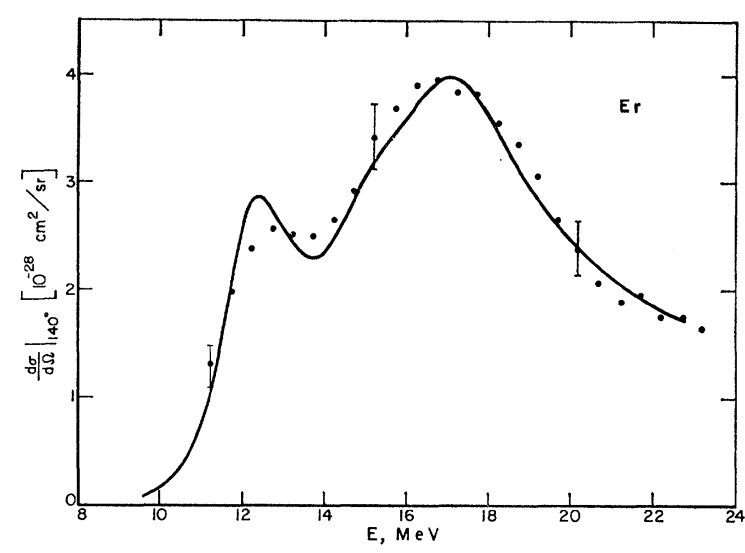

FIg. 7. Total quasi-elastic scattering cross section of Er with experimental data from Ref. 21, multiplied by $7 / 9$.

indicated. This would yield for the $\eta$-vibration parameter about $E_{\gamma} \approx 500-700 \mathrm{keV}$. Such a value for $E_{\gamma}$ would also agree with the systematics of the $\eta$-vibrational energies in the neighboring nuclei. ${ }^{24}$ Because of these uncertainties, we took $E_{\gamma}$ to be a free parameter.

TABLE II. Resonance parameters for ${ }^{165} \mathrm{Ho}$.

\begin{tabular}{ccccccccc}
\hline \hline $\begin{array}{c}E_{R} \\
(\mathrm{keV})\end{array}$ & $\begin{array}{c}E_{\gamma} \\
(\mathrm{MeV})\end{array}$ & $\begin{array}{c}E_{\beta} \\
(\mathrm{MeV})\end{array}$ & $\begin{array}{c}E_{0} \\
(\mathrm{MeV})\end{array}$ & $\begin{array}{c}\Gamma_{0} \\
(\mathrm{MeV})\end{array}$ & $\beta_{0}$ & $\delta$ & $\alpha$ & Ref. \\
\hline 10.5 & 0.6 & 1.46 & 12.0 & 2.1 & 0.28 & 1.5 & 0.05 & 19 \\
10.5 & 1.0 & 1.46 & 12.0 & 2.3 & 0.24 & 1.6 & 0.13 & 25
\end{tabular}

Two absorption experiments exist in the literature. ${ }^{19,25}$ We matched theoretical absorption cross sections to both sets of experimental data. The parameters obtained are listed in Table II. ${ }^{20}$

The cross section corresponding to the data of Ref. 25 is shown in Fig. 8. These data suggest the onset of the

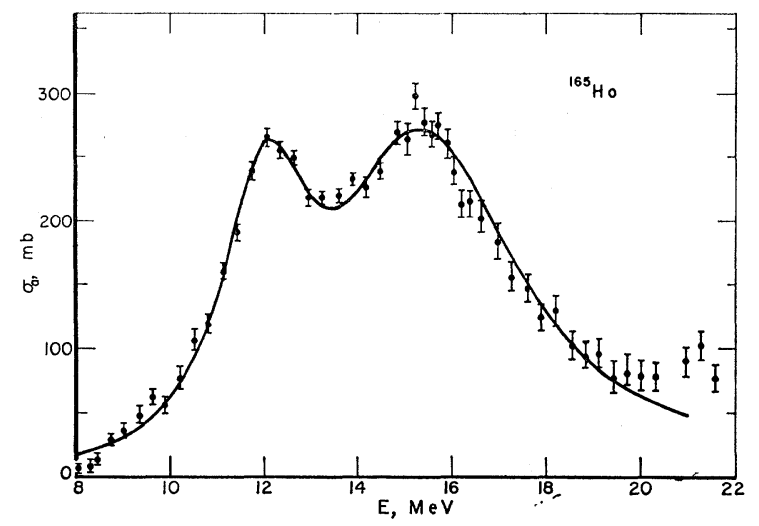

FIG. 8. $\gamma$-absorption cross section of ${ }^{165} \mathrm{Ho}$ from Ref. 25. The theoretical curve is computed with the second parameter set of Table II.

${ }^{24}$ A. Faessler, W. Greiner, and R. K. Sheline, Nucl. Phys. 70, 33 (1965).

${ }_{25}$ R. L. Bramblett, J. T. Caldwell, G. F. Auchampaugh, and S. C. Fultz, Phys. Rev. 129, 2723 (1963). 


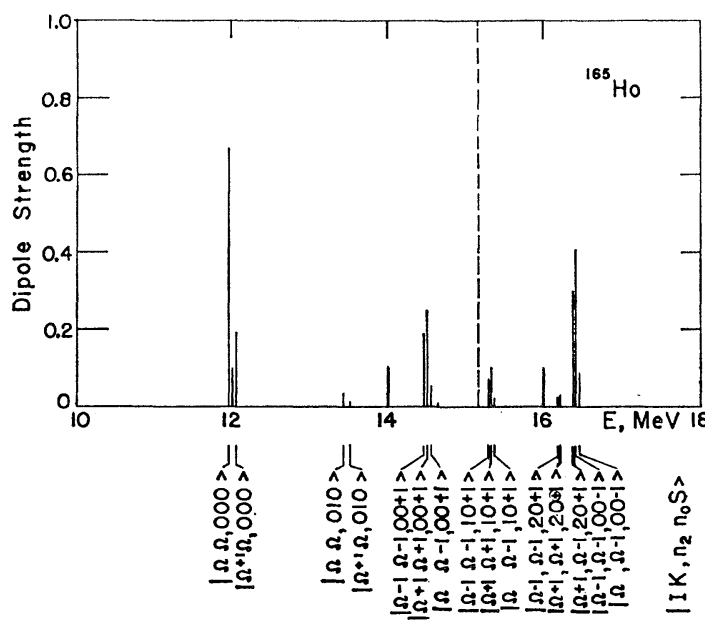

Fig. 9. Level scheme and dipole strengths of the giant dipole resonance states of ${ }^{165} \mathrm{Ho}$.

E2 giant resonance at about $21 \mathrm{MeV}^{6,26}$ The cross section corresponding to the data of Ref. 19 is not shown separately, since it is practically indistinguishable from Fig. 1.

The spectrum and the strengths of the dipole states which are computed with the first parameter set of Table II are shown in Fig. 9. The spectrum now is considerably richer. However, the dipole-strength distribution has remained practically unchanged compared to the even-even case. Thus, summing the strengths of the different main peaks, we have the distribution 86:51:20:79 for ${ }^{165} \mathrm{Ho}$ as compared to 86:58:17:80 for ${ }^{166} \mathrm{Er}$. This is a quantitative test for the assumption that the odd particle has no influence on the distribution of the dipole strengths. However, the dipole strength is split up into several components lying at exceedingly close energies.

The different contributions to the scattering cross section are shown in Figs, 10-13. Because of the finite

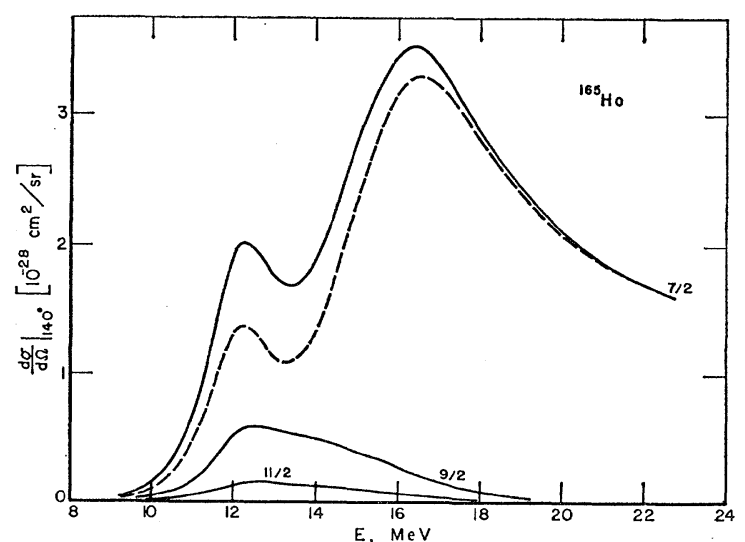

FIG. 10. Calculated elastic and inelastic scattering cross sections for scattering into the ground-state band of ${ }^{165} \mathrm{Ho}$.

${ }^{26}$ R. Ligensa and W. Greiner, Nucl. Phys. A92, 673 (1967). ground-state spin, the elastic scattering now has both scalar tensor contributions. The scalar contribution is indicated separately by the dashed line in Fig. 10. Now two rotational Raman lines of the ground-state band can be reached. They are also shown in Fig. 10. Figure 11 shows the Raman scattering into the states of the $\gamma$ bands. In the lower $\gamma$ band $\left(K=\frac{3}{2}\right)$ five rotational states can be reached. The corresponding scattering cross sections are plotted except for the transition into the state $I=11 / 2, K=\frac{3}{2}$, which has too small an intensity to be plotted. Because of the dipole selection rules only the band head of the $K=11 / 2 \gamma$ band can be reached. The total scattering into the $\gamma$ bands corresponds to the scattering into the $\gamma$-band head in even-even nuclei as discussed in detail above.

The Raman scattering into the $\beta$ band and the higher $\gamma$ band are shown in Figs. 12 and 13. The total quasi-elastic scattering cross section is shown in Fig. 14, together with the available experimental data..$^{21,27}$

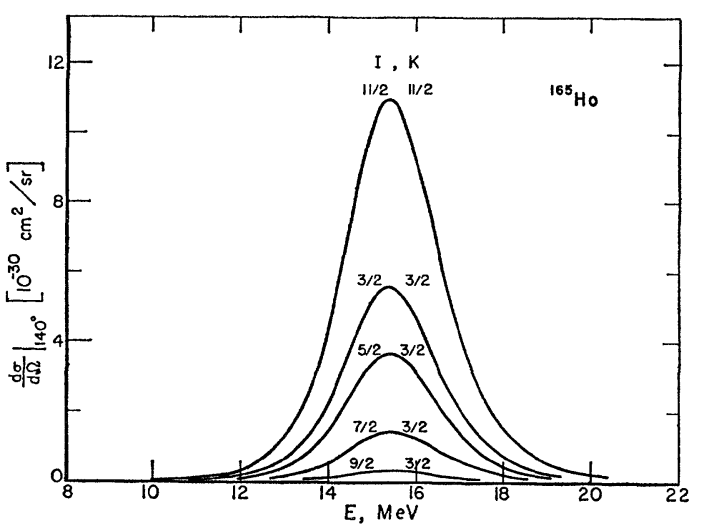

FIG. 11. Calculated inelastic scattering cross sections for scattering into the two $\gamma$ bands of ${ }^{165} \mathrm{Ho}$.

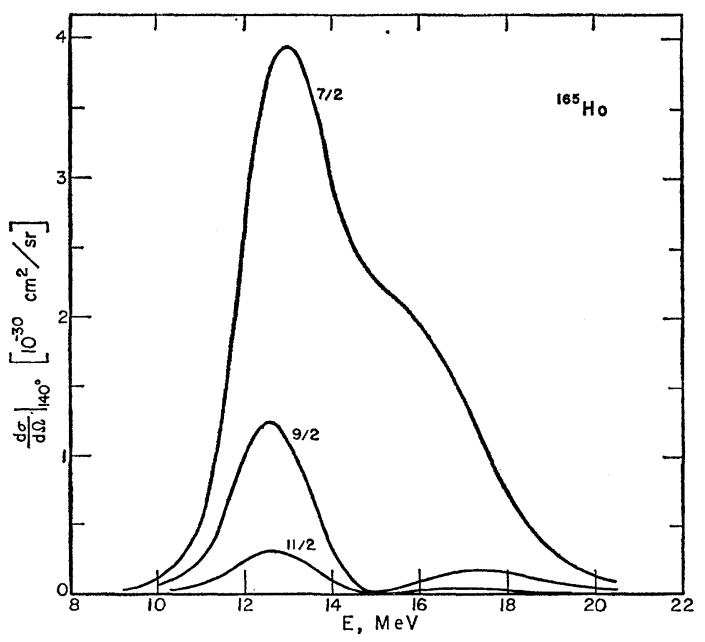

Fig. 12. Calculated inelastic scattering cross sections for scattering into the $\beta$ band of ${ }^{165} \mathrm{Ho}$.

${ }^{27}$ P. A. Tipler, P. Axel, N. Stein, and D. C. Sutton, Phys. Rev. 129, 2096 (1963). 
The quasi-elastic scattering cross section computed with the parameters obtained from the fit of the absorption cross section to the Fuller-Hayward data ${ }^{19,20}$ is shown in Fig. 15. Both sets of parameters evidently give reasonable agreement with the scattering data. However, considering the total mass of data together, the Fuller-Hayward data give a more consistent over-all picture. Firstly, the parameter $E_{\gamma}$ is more in line with the value expected from the low-energy spectrum (see above). The same holds for the deformation parameter $\beta_{0}$, which in this region of atomic number is around 0.3 instead of 0.24 . Finally, the agreement with the scattering data of the two theoretical curves seem to favor somewhat the Fuller-Hayward parameters. We believe that two systematic effects are responsible for the differences between the betatron data ${ }^{19}$ and the positronannihilation data..$^{25}$ First, it seems that the resolution of the betatron experiment is higher. This shows up the difference in the values $\Gamma_{0}$, viz., 2.1 and $2.3 \mathrm{MeV}$. Second, the neutron multiplicity corrections seem to have been overestimated by the Livermore group. All

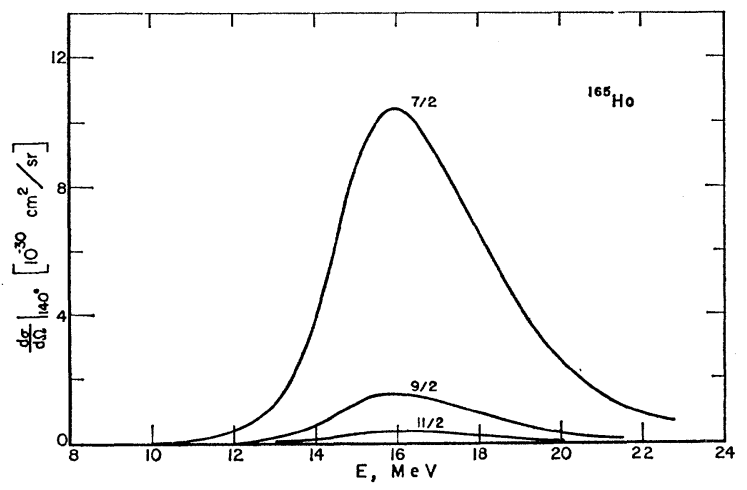

FIG. 13. Calculated inelastic scattering cross sections for scattering into the higher $\gamma$ band of ${ }^{165} \mathrm{Ho}$.

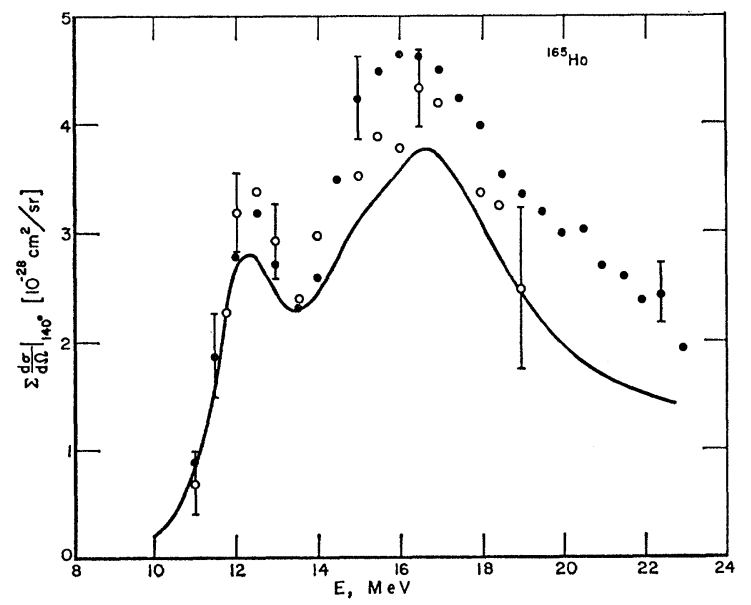

FIG. 14. Total quasi-elastic scattering cross section of ${ }^{165} \mathrm{Ho}$ from Ref. 27 (open circles) and Ref. 21 (closed circles multiplied by $7 / 9)$. The theoretical curve is computed with the second set of parameters of Table II.

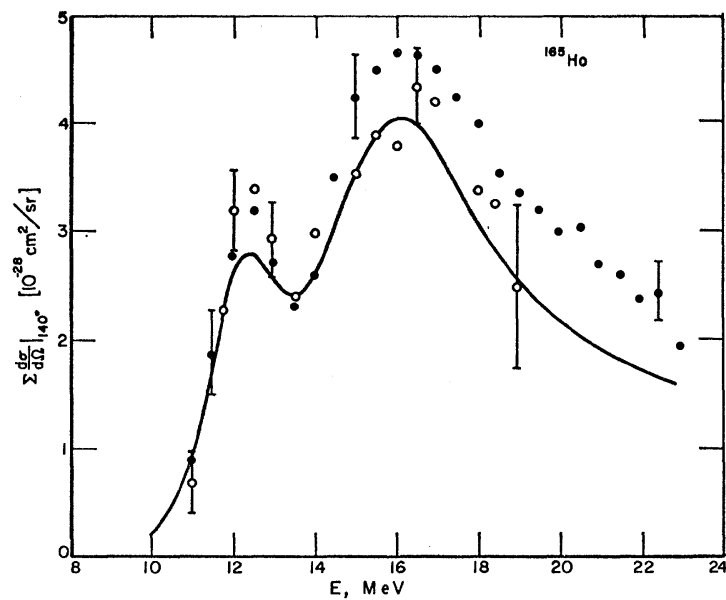

Fig. 15. Total quasi-elastic scattering cross section of ${ }^{165} \mathrm{Ho}$ computed with the first set of parameters of Table II, except $\alpha=0.16$; experimental points as in Fig. 14 .

these discrepancies lie within the stated systematic uncertainties of the experiments. Each of our stated reasons by itself would not be sufficient to favor one set of data over the other. However, taken together, we believe that they justify our conclusion.

\section{Terbium}

Measurements on ${ }^{159} \mathrm{~Tb}$ have been performed by various groups, three of which were available to us. ${ }^{28-30}$ The obtained resonance parameters are given in Table III.

$E_{\gamma}$ is in good agreement with those of neighboring nuclei. However, the deformation parameter $\beta_{0}$ is in both cases smaller than that obtained from Coulomb excitation.

Both sets of parameters give reasonable fits, as Figs. 16 and 17 show. The total scattering cross sections are given in Figs. 18 and 19. The scattering data are those of Ref. 21, except that they are multiplied by 0.823 and shifted in energy in the plots of Figs. 18 and 19.

\section{Tantalum}

For ${ }^{181} \mathrm{Ta}$ we used the data of three groups, ${ }^{25,28,29}$ two of which coincide within the experimental errors. ${ }^{25,29}$

TABLE III. Resonance parameters of ${ }^{159} \mathrm{~Tb}$.

\begin{tabular}{ccccccccc}
\hline \hline $\begin{array}{c}E_{R} \\
(\mathrm{keV})\end{array}$ & $\begin{array}{c}E_{\gamma} \\
(\mathrm{MeV})\end{array}$ & $\begin{array}{c}E_{\beta} \\
(\mathrm{MeV})\end{array}$ & $\begin{array}{c}E_{0} \\
(\mathrm{MeV})\end{array}$ & $\begin{array}{c}\Gamma_{0} \\
(\mathrm{MeV})\end{array}$ & $\beta_{0}$ & $\delta$ & $\alpha$ & Ref. \\
\hline 11.6 & 1.0 & 1.5 & 12.00 & 2.5 & 0.24 & 1.6 & 0.17 & 30 \\
11.6 & 1.0 & 1.5 & 12.34 & 2.7 & 0.26 & 1.4 & 0.68 & 28,29 \\
\hline
\end{tabular}

${ }^{28}$ E. G. Fuller and M. S. Weiss, Phys. Rev. 112, 560 (1958).

${ }_{29}$ O. V. Bogdankevich, B. I. Goryachev, and V. A. Zapevalov, Zh. Eksperim. i Teor. Fiz. 42, 1502 (1962) [English transl.: Soviet Phys.-JETP 15, 1044 (1962)].

${ }_{30}$ R. L. Bramblett, J. T. Caldwell, R. R. Harvey, and S. C. Fultz, Phys. Rev. 133, B869 (1964). 


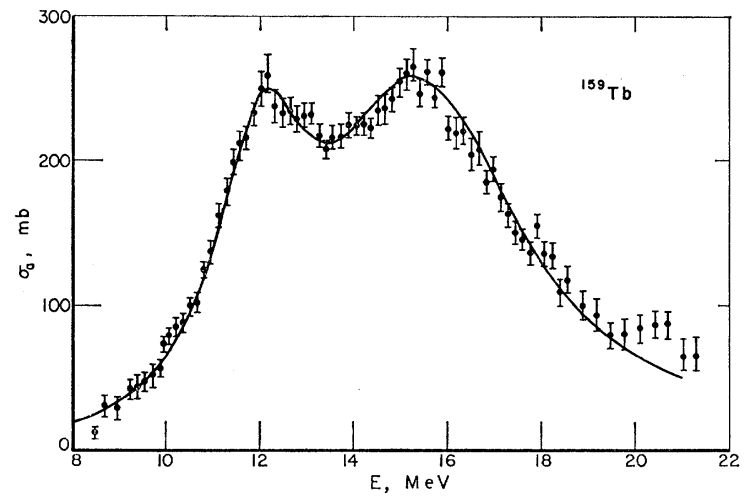

FIG. 16. $\gamma$-absorption cross section of ${ }^{159} \mathrm{~Tb}$ from Ref. 30. The theoretical curve is computed with the first set of parameters of Table III.

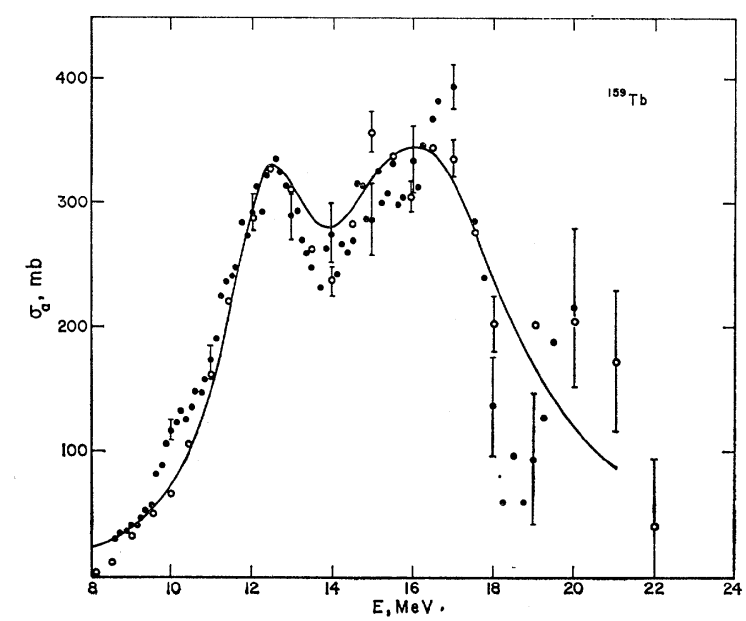

FIG. 17. $\gamma$-absorption cross section of ${ }^{159} \mathrm{~Tb}$ from Ref. 28 (circles, multiplied by 1.07) and from Ref. 29 (dots). The theoretical curve is computed with the second set of parameters of Table III.

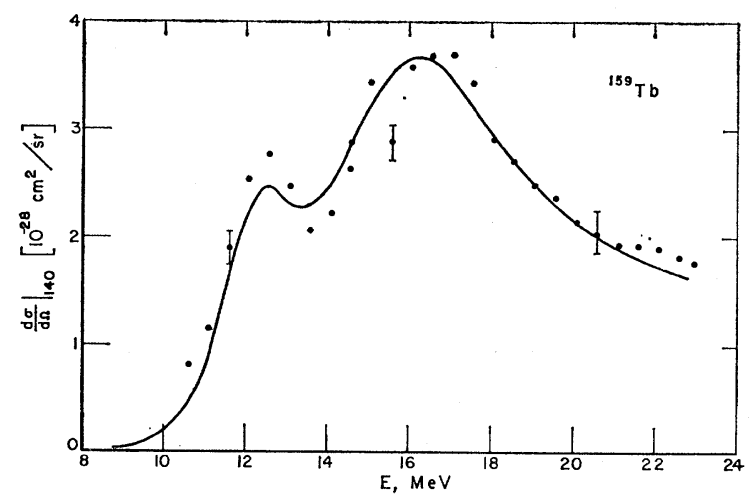

Fig. 18. Total quasi-elastic scattering cross section of ${ }^{159} \mathrm{~Tb}$ from Ref. 21 (multiplied by 0.823 ; energy scale is shifted up by $0.6 \mathrm{MeV}$ ). The theoretical curve is computed with the first set of parameters of Table III.

The absorption cross sections are shown in Figs. 20 and 21 and the fitting parameters are listed in Table IV.

The fitting parameters differ in the values for $E_{\gamma}$ and

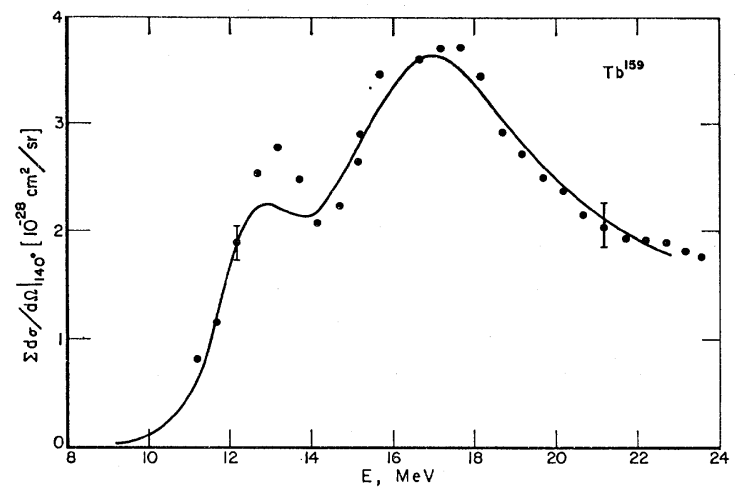

FIG. 19. Total quasi-elastic scattering cross section of ${ }^{159} \mathrm{~Tb}$ computed with the second set of parameters of Table III, except $\alpha=0.175$; experimental points as in Fig. 18 .

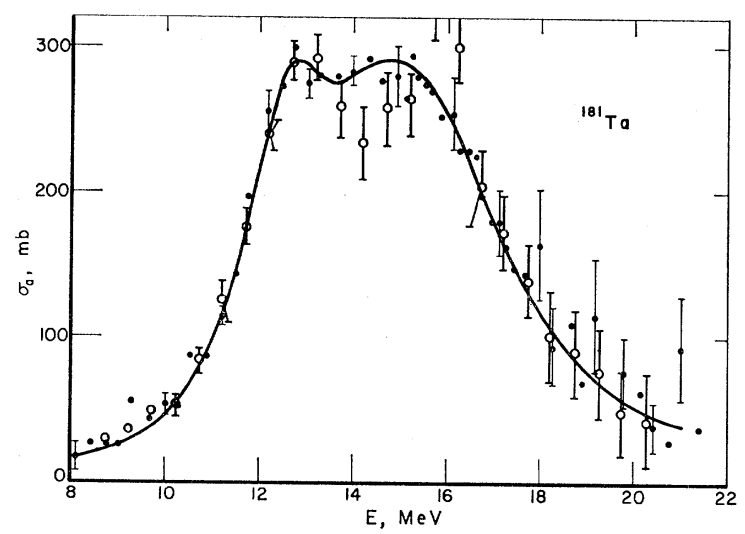

FIG. 20. $\gamma$-absorption cross section of ${ }^{181}$ Ta from Refs. 25 and 29. (Data from Ref. 29 is multiplied by 0.652 .) The theoretical curve is computed with the first set of parameters of Table IV.

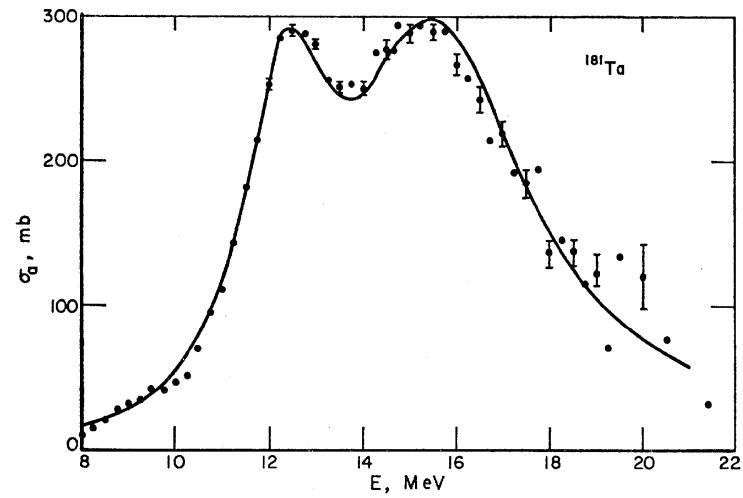

Fig. 21. $\gamma$-absorption cross section of ${ }^{181}$ Ta from Ref. 28 (multiplied by 0.704 ). The theoretical curve is computed with the second set of parameters of Table IV.

slightly for $E_{0}$. In this region one would expect $E_{\gamma} \approx 1.2$ $\mathrm{MeV}, \beta_{0} \approx 0.20-0.25$ from neighboring nuclei. The resulting total scattering cross sections in Figs. 22 and 23 are both in reasonable agreement with the experimental data of Ref. 21. 


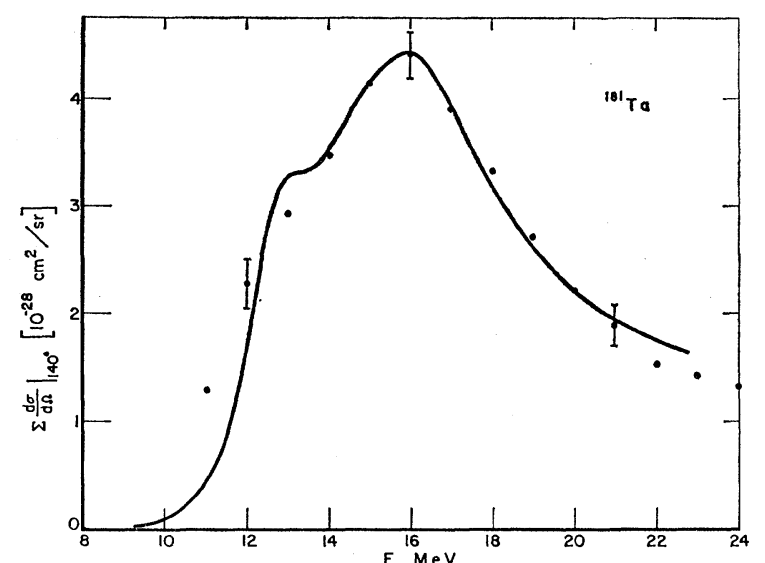

FIG. 22. Total quasi-elastic scattering cross section of ${ }^{181} \mathrm{Ta}$ from Ref. 21 (multiplied by 0.65 ; energy scale is shifted up by $1.0 \mathrm{MeV}$ ). The theoretical curve is computed with the first set of parameters of Table IV.

\section{SUMMARY}

In this paper we have found good agreement between experimental data and theoretical predictions. The obtained nuclear parameters were found to be consistent with the values expected from low-energy spectra. Also, the data on the damping parameters of the giant resonance $\Gamma_{0}$ and $\delta$ are consistent with theoretical estimates. ${ }^{10}$

However, there seems to be an indication that the

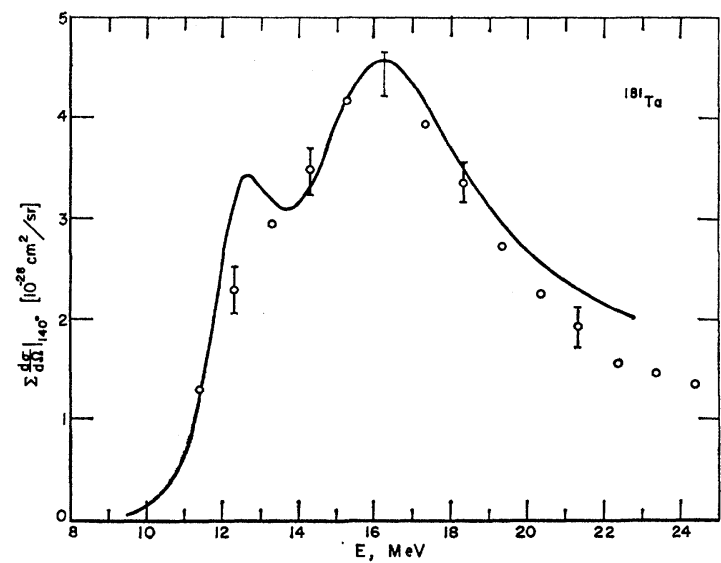

Fig. 23. Total quasi-elastic scattering cross section of ${ }^{181} \mathrm{Ta}$ computed with the second set of parameters of Table IV. Experimental points as in Fig. 22 (energy scale is shifted up by $1.2 \mathrm{MeV}$ ).

TABLE IV. Resonance parameters for ${ }^{181} \mathrm{Ta}$.

\begin{tabular}{ccccccccc}
\hline \hline $\begin{array}{c}E_{R} \\
(\mathrm{keV})\end{array}$ & $\begin{array}{c}E_{\gamma} \\
(\mathrm{MeV})\end{array}$ & $\begin{array}{c}E_{\beta} \\
(\mathrm{MeV})\end{array}$ & $\begin{array}{c}E_{0} \\
(\mathrm{MeV})\end{array}$ & $\begin{array}{c}\Gamma_{0} \\
(\mathrm{MeV})\end{array}$ & $\beta_{0}$ & $\delta$ & $\alpha$ & Ref. \\
\hline 15.1 & 0.6 & 1.4 & 12.55 & 2.3 & 0.19 & 1.8 & 0.03 & 25,29 \\
15.1 & 1.2 & 1.4 & 12.35 & 2.3 & 0.21 & 1.8 & 0.12 & 28 \\
\hline
\end{tabular}

"high-energy deformations" are slightly smaller than the measured $\beta_{0}$ 's from the low-energy spectrum, as shown in Table V. ${ }^{31}$

TABLE V. The deformation parameter $\beta_{0}$ of ${ }^{159} \mathrm{~Tb},{ }^{165} \mathrm{Ho},{ }^{166} \mathrm{Er},{ }^{181} \mathrm{Ta}$, and neighboring even-even nuclei from Coulomb excitation $\left(\beta_{0, \mathrm{CE}}\right)$ and from the giant-resonance splitting $\left(\beta_{0, \mathrm{GR}}\right)$. The $B(E 2)$ values are taken from Ref. $31 . \beta_{0, \mathrm{CE}}$ is evaluated from

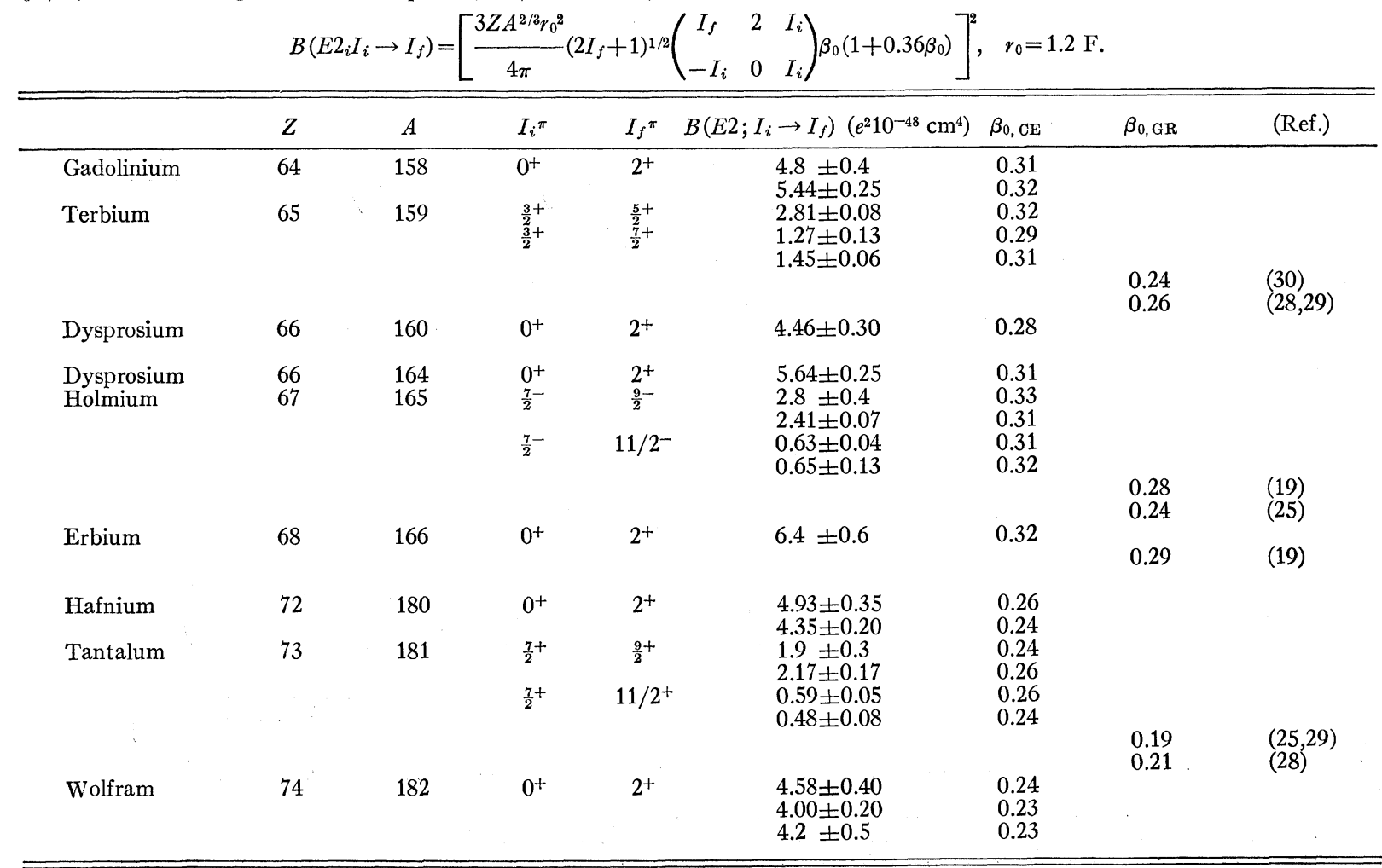


The deformation parameter $\beta_{0, \mathrm{CE}}$ for odd $-A$ nuclei which are deduced from Coulomb excitation data are in good agreement with those of neighboring even-even nuclei. This is expected from the collective property of $\beta_{0}$. In all cases, $\beta_{0, \mathrm{GR}}$ taken from the giant resonance is smaller than $\beta_{0, \mathrm{CE}}$. More precise absorption and scattering measurements are required to show whether these indications are indeed true.

Some of these discrepancies may be associated with the incompleteness of the employed model. First, of the nuclear surface modes only the quadrupole mode has been treated dynamically, and higher multipoles of the nuclear deformation have been neglected. Even limiting oneself to terms containing at most three amplitude functions, evidently a large number of couplings with the higher multipole modes are possible, e.g., $b^{(1) \dagger b^{(2)}} \alpha^{(3) \dagger}$, where $\alpha^{(3) \dagger}$ is the creation operator for surface octupole oscillations. Also, the higher static deformations ${ }^{32}$ can have an influence on the results.

The model also does not yet incorporate the lowenergy tails of the nonresonating high-energy absorption mechanisms, viz., the direct photo-ionization processes and the quasideuteron effect. The existence of these effects is suggested by the photon-absorption experiments of Ambler, Fuller, and Marshak ${ }^{8}$ with aligned nuclei. Unfortunately, the experiments with nonaligned targets are not sufficiently accurate to show the difference in the shape of various cross sections which would result from the presence of an additional scalar component with a magnitude of about $15 \%$ indicated by the experiments, ${ }^{8}$ a magnitude consistent with theoretical expectations about these nonresonating processes.

The small irregularities on the rising side of the cross section are very likely the effects of the individual particle structure which in the Brown-Bolsterli model ${ }^{33}$ would give up all the dipole strengths to the collective states. Such states, perhaps, can be described in the collective model by spin-isospin waves first considered by Wild. ${ }^{34}$

The modifications of the predictions which would arise if the theory would be refined to take into account these effects can be expected to be small. Within these limitations, agreement between theory and experiment is such that one has to conclude that the collective model is valid to a very high degree for nuclei of the deformed region.

\section{ACKNOWLEDGMENTS}

We would like to thank E. G. Fuller for fruitful discussions and R. Ligensa for the calculation of the quadrupole matrix elements.

\section{APPENDIX}

We write Eq. (9) in the case of only electric-dipole and -quadrupole radiation:

$$
K_{i f}=(-)^{p^{\prime}} \sum_{\substack{L=1 \\ M}}^{2} R_{p M^{L} \mathfrak{D}_{M,-p^{\prime}} L}
$$

with

$$
\begin{aligned}
R_{p M}{ }^{L}=(-)^{L} \sum_{n}\left(C_{1 n}{ }^{L L}\left\langle f\left|\int \mathbf{j} \cdot \mathbf{A}_{L M^{k}}(e)\right| n\right\rangle\left\langle n\left|\int \mathbf{j} \cdot \mathbf{A}_{L p}{ }^{k}(e)\right| i\right\rangle\right. & \\
& \left.+C_{2 n}{ }^{L L}\left\langle f\left|\int \mathbf{j} \cdot \mathbf{A}_{L p}{ }^{k}(e)\right| n\right\rangle\left\langle n\left|\int \mathbf{j} \cdot \mathbf{A}_{L M^{k}}(e)\right| i\right\rangle\right)-(-)^{p} \delta_{L 1} \boldsymbol{\delta}_{i f} \frac{Z^{2} e}{A M C^{2}} .
\end{aligned}
$$

The decomposition of $R_{p M^{L}}$ into irreducible parts $\Re_{L^{\prime} M^{\prime}}{ }^{L}$ leads to

with

$$
R_{p M}{ }^{L}=\sum_{L^{\prime}=0}^{2 L}(-)^{M+p}\left(2 L^{\prime}+1\right)^{1 / 2}\left(\begin{array}{ccc}
L & L & L^{\prime} \\
p & M & -M-p
\end{array}\right) \Re_{L^{\prime} M+p}{ }^{L},
$$

$$
\begin{aligned}
& \Re_{L^{\prime} M^{\prime}} L=\sum_{p}(-)^{M^{\prime}}\left(2 L^{\prime}+1\right)^{1 / 2}\left(\begin{array}{ccc}
L & L & L^{\prime} \\
p & M^{\prime}-p & -M^{\prime}
\end{array}\right) R_{p, M^{\prime}-p^{L}} \\
& =\sum_{p}(-)^{L+M^{\prime}}\left(2 L^{\prime}+1\right)^{1 / 2}\left(\begin{array}{ccc}
L & L & L^{\prime} \\
p & M^{\prime}-p & -M^{\prime}
\end{array}\right)\left\{\sum_{n}(-)^{I_{f}-M_{f}+I_{n}-M_{n}}\right. \\
& \times\left[\left(\begin{array}{ccc}
I_{f} & L & I_{n} \\
-M_{f} & M^{\prime}-p & M_{n}
\end{array}\right)\left(\begin{array}{ccc}
I_{n} & L & I_{i} \\
-M_{n} & p & M_{i}
\end{array}\right) \mathfrak{S}_{1 n}{ }^{L}+\left(\begin{array}{ccc}
I_{f} & L & I_{n} \\
-M_{f} & p & M_{n}
\end{array}\right)\left(\begin{array}{ccc}
I_{n} & L & I_{i} \\
-M_{n} & M^{\prime}-p & M_{i}
\end{array}\right) \mathfrak{S}_{2 n}{ }^{L}\right] \\
& \left.+\delta_{L 1} \delta_{i f}(-)^{p} \frac{Z^{2} e^{2}}{A M c^{2}}\right\}
\end{aligned}
$$

${ }^{31} \mathrm{~J}$. Lindskog, T. Sundstrom, and P. Sparmann, in Alpha-Beta- and Gamma-Ray Spectroscopy, edited by K. Siegbahn (Interscience Publishers, Inc., New York, 1965), Vol. II.

${ }^{33}$ G. E. Brown and M. Bolsterli, Phys. Rev. Letters 3, 472 (1959).

${ }^{34} \mathrm{~W}$. Wild, Sitzber, Math.-Naturw. Kl. Bayer. Akad. Wiss. Muenchen 1955, 371 (1955).

${ }^{32}$ M. Danos, W. Greiner, and C. B. Kohr, Phys. Letters 12, 344 (1964). 
using the Wigner-Eckart theorem (14) for the photon-interaction matrix elements. The meaning of the $\mathcal{E}_{i n}{ }^{L}$ is given in (13). $M^{\prime}$ is restricted to $M^{\prime}=M_{f}-M_{i}$. The sum of three $3 j$ symbols over $p$ shortens to a $3 j$ and a $6 j$ symbol.

$$
\begin{aligned}
& \sum_{p}(-)^{I_{n}-M_{n}-M^{\prime}}\left(\begin{array}{ccc}
L & L & L \\
p & M^{\prime}-p & -M^{\prime}
\end{array}\right)\left(\begin{array}{ccc}
I_{f} & L & I_{n} \\
-M_{f} & M^{\prime}-p & M_{n}
\end{array}\right)\left(\begin{array}{ccc}
I_{n} & L & I_{i} \\
-M_{n} & p & M_{i}
\end{array}\right)=(-)^{L^{\prime}}\left(\begin{array}{ccc}
L^{\prime} & I_{f} & I_{i} \\
M^{\prime} & -M_{f} & M_{i}
\end{array}\right)\left\{\begin{array}{lll}
L^{\prime} & I_{f} & I_{i} \\
I_{n} & L & L
\end{array}\right\}, \\
& \sum_{p}(-)^{I_{n}-M_{n}-M^{\prime}}\left(\begin{array}{ccc}
L & L & L^{\prime} \\
p & M^{\prime}-p & -M^{\prime}
\end{array}\right)\left(\begin{array}{ccc}
I_{f} & L & I_{n} \\
-M_{f} & p & M_{n}
\end{array}\right)\left(\begin{array}{ccc}
I_{n} & L & I_{i} \\
-M_{n} & M^{\prime}-p & M_{i}
\end{array}\right) \\
&=\left(\begin{array}{ccc}
L^{\prime} & I_{f} & I_{i} \\
M^{\prime} & -M_{f} & M_{i}
\end{array}\right)\left\{\begin{array}{lll}
L^{\prime} & I_{f} & I_{i} \\
I_{n} & L & L
\end{array}\right\} .
\end{aligned}
$$

If $i=f$, then $M^{\prime}=0$, and

$$
\sum_{p}(-)^{p}\left(\begin{array}{ccc}
L & L & L^{\prime} \\
p & -p & 0
\end{array}\right)=-\sqrt{3} \delta_{L^{\prime} 0}, \quad\left(\begin{array}{ccc}
0 & I_{i} & I_{i} \\
0 & -M_{i} & M_{i}
\end{array}\right)=(-)^{I_{i}-M_{i}}(-)^{2 I_{i}}\left(2 I_{i}+1\right)^{-1 / 2}
$$

Inserting (A5) and (A6) in (A4), we get

$$
\begin{aligned}
& \Re_{L^{\prime} M^{\prime}} L^{2}=(-)^{I_{f}-M_{f}}\left(\begin{array}{ccc}
L^{\prime} & I_{f} & I_{i} \\
M^{\prime} & -M_{f} & M_{i}
\end{array}\right)\left(2 L^{\prime}+1\right)^{1 / 2}
\end{aligned}
$$

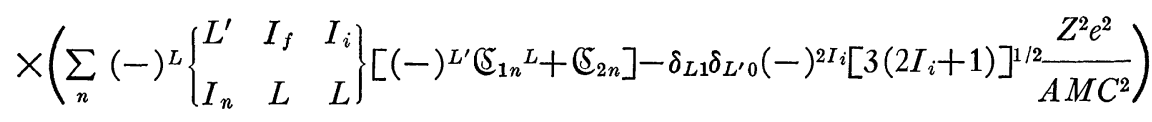

$$
\begin{aligned}
& =(-)^{I_{f}-M_{f}}\left(2 L^{\prime}+1\right)^{1 / 2}\left(\begin{array}{ccc}
L^{\prime} & I_{f} & I_{i} \\
M^{\prime} & -M_{f} & M_{i}
\end{array}\right) \Re_{L^{\prime}}{ }^{L} .
\end{aligned}
$$

Therefore we get from (A1), (A3), and (A7)

$$
K_{i f}=(-)^{p^{\prime}} \sum_{L=1}^{2} \sum_{L^{\prime}=0}^{2 L}(-)^{I f-M_{i}}\left(2 L^{\prime}+1\right)\left(\begin{array}{ccc}
L & L & L^{\prime} \\
p & M & -M-p
\end{array}\right)\left(\begin{array}{ccc}
L^{\prime} & I_{f} & I_{i} \\
M+p & -M_{f} & M_{i}
\end{array}\right) \Re_{L^{\prime}} L_{D_{M},-p^{\prime}}{ }^{L} .
$$

This is Eq. (11) with the polarizabilities (12). 